\title{
Top-down, knowledge-based genetic reduction of yeast central carbon metabolism
}

Eline D. Postma ${ }^{1}$, Lucas G.F. Couwenberg ${ }^{1}$, Roderick N. van Roosmalen ${ }^{1}$, Jordi Geelhoed ${ }^{1}$, Philip A. de Groot $^{1}$, Pascale Daran-Lapujade ${ }^{1^{*}}$

${ }^{1}$ Department of Biotechnology, Delft University of Technology, van der Maasweg 9, 2627HZ Delft, The Netherlands

${ }^{*}$ Corresponding author:

Tel: +31 152789965

Email: p.a.s.daran-lapujade@tudelft.nl

Keywords: Saccharomyces cerevisiae, central carbon metabolism, minimal genome, genetic redundancy 


\section{Abbreviations}

\begin{tabular}{|c|c|c|c|}
\hline CCM & Central Carbon Metabolism & RL5P & Ribulose 5-phosphate \\
\hline WGD & Whole Genome Duplication & R5P & Ribose 5-phosphate \\
\hline SSD & Smaller-Scale Duplication & XUL-5P & Xylulose 5-phosphate \\
\hline MG & Minimal Glycolysis & S7P & Sedoheptulose 7-phosphate \\
\hline PPP & Pentose Phosphate Pathway & Ery-4P & Erythrose 4-phosphate \\
\hline $\mathrm{MC}$ & Mitochondrial Carrier & OAA & Oxaloacetate \\
\hline TCA cycle & Tricarboxylic Acid cycle & Cit & Citrate \\
\hline glyc $^{\min }$ & minimized glycolysis & Cis-Aco & Cis-aconitate \\
\hline \multirow[t]{2}{*}{ fer $r^{\min }$} & minimized ethanolic & Isocit & Isocitrate \\
\hline & fermentation & $\alpha-K G$ & $\alpha$-ketoglutarate \\
\hline \multirow[t]{2}{*}{$\mathrm{ppp}^{\min }$} & minimized pentose phosphate & Suc-CoA & Succinyl-CoA \\
\hline & pathway & Suc & Succinate \\
\hline \multirow[t]{2}{*}{ tcamin } & minimized tricarboxylic acid & Fum & Fumarate \\
\hline & cycle & Mal & Malate \\
\hline \multirow[t]{2}{*}{$\mathrm{mc}^{\mathrm{min}}$} & minimized mitochondrial & Suc & Succinate \\
\hline & carriers & Glyox & Glyoxylate \\
\hline fum $^{\min }$ & minimized fumarate reductases & Ace & Acetate \\
\hline glyox ${ }^{\min }$ & minimized glyoxylate cycle & Ac-CoA & Acetyl-CoA \\
\hline$a_{c e} \min$ & minimized Acetyl-CoA synthesis & Glyc-3P & Glycerol-3-phosphate \\
\hline glycerol $^{\min }$ & minimized glycerol synthesis & YPD & Yeast Peptone Dextrose \\
\hline Glc & Glucose & SMD & Synthetic Medium Dextrose \\
\hline Glc-6P & Glucose-6-phosphate & SME & Synthetic Medium Ethanol \\
\hline Fru-6P & Fructose-6-phosphate & SMGal & Synthetic Medium Galactose \\
\hline Fru-1,6-BP & Fructose-1,6-bisphosphate & SMMal & Synthetic Medium Maltose \\
\hline GAP & Glyceraldehyde-3-phosphate & SMSuc & Synthetic Medium Sucrose \\
\hline DHAP & Dihydroxyacetone phosphate & SMFruc & Synthetic Medium Fructose \\
\hline 1,3-BPG & 1,3-bisphosphoglycerate & SMPyr & Synthetic Medium Pyruvate \\
\hline 3-PG & 3-phosphoglycerate & & \\
\hline 2-PG & 2-phosphoglycerate & & \\
\hline PEP & Phosphoenolpyruvate & & \\
\hline Pyr & Pyruvate & & \\
\hline AcAl & Acetaldehyde & & \\
\hline EtOH & Ethanol & & \\
\hline 6P-GLCN-lac & 6-Phosphogluconolactone & & \\
\hline 6P-GLCN & 6-Phosphoglucononate & & \\
\hline
\end{tabular}




\section{Abstract}

Saccharomyces cerevisiae, whose evolutionary past includes a whole-genome duplication event, is characterised by a mosaic genome configuration with substantial apparent genetic redundancy. This apparent redundancy raises questions about the evolutionary driving force for genomic fixation of 'minor' paralogs and complicates modular and combinatorial metabolic engineering strategies. While isoenzymes might be important in specific environments, they could be dispensable in controlled laboratory or industrial contexts. The present study explores the extent to which the genetic complexity of the central carbon metabolism (CCM) in S. cerevisiae, here defined as the combination of glycolysis, pentose phosphate pathway, tricarboxylic acid cycle and a limited number of related pathways and reactions, can be reduced by elimination of (iso)enzymes without major negative impacts on strain physiology. Cas9mediated, groupwise deletion of 35 from the 111 genes yielded a 'minimal CCM' strain, which despite the elimination of $32 \%$ of CCM-related proteins, showed only a minimal change in phenotype on glucosecontaining synthetic medium in controlled bioreactor cultures relative to a congenic reference strain. Analysis under a wide range of other growth and stress conditions revealed remarkably few phenotypic changes of the reduction of genetic complexity. Still, a well-documented context-dependent role of GPD1 in osmotolerance was confirmed. The minimal CCM strain provides a model system for further research into genetic redundancy of yeast genes and a platform for strategies aimed at large-scale, combinatorial remodelling of yeast CCM. 


\section{Introduction}

The fundamental challenge of defining the minimum complement of genes required for life has been addressed by theoretical as well as experimental approaches. Bottom-up and top-down strategies mainly focused on bacteria with small genomes (Hashimoto et al. 2005; Hutchison et al. 2016; Komatsu et al. 2010; Lara and Gosset 2019; Lieder et al. 2015; Morimoto et al. 2008; Mutschler et al. 2019; Suzuki et al. 2005; Zhu et al. 2017). Their larger genome sizes might appear to make eukaryotic microorganisms less relevant for this type of research. However, they do offer attractive models to explore the biological significance of (apparent) genetic redundancy. Different evolutionary advantages have been proposed for the fixation of duplicated genes in genomes, including provision of a molecular landscape for functional (minor or major) innovation (e.g., neo- and subfunctionalization), a functional backup, gene dosage effects or increased buffering to respond to environmental cues (Conant and Wolfe 2008; Escalera-Fanjul et al. 2019). Systematically identifying the physiological significance underlying gene fixation presents a daunting challenge.

With its relatively small genome (12 Mb), tractability and high genetic accessibility, the yeast Saccharomyces cerevisiae is a valuable model for fundamental research on minimal genetic requirements. S. cerevisiae underwent a whole genome duplication (WGD) approximately 100 million years ago as well as Smaller-Scale Duplication (SSD) events. While $90 \%$ of the WGD genes were lost during evolution, some duplicates remain (Escalera-Fanjul et al. 2019). As in humans, a substantial fraction of the total gene duplicates in S. cerevisiae originates from the WGD (approximately $63 \%$ of duplicates in S. cerevisiae genome and $62 \%$ in the human genome), while a smaller fraction originates from SSD (approximately 37 $\%$ of duplicates in S. cerevisiae genome and $38 \%$ in the human genome) (Acharya and Ghosh 2016; Fares et al. 2013). While systematic, large scale studies like the construction of the yeast deletion collections (Costanzo et al. 2016; Giaever et al. 2002; Kuzmin et al. 2018; Winzeler et al. 1999), the synthetic genetic array projects (Costanzo et al. 2010; Tong et al. 2001; Tong et al. 2004) or the recent SCRaMbLE-based genome compaction (Luo et al. 2021), have provided valuable information on the dispensability of (a combination of) genes, the physiological role of many of these paralogous genes remains poorly defined.

In addition to the fundamental scientific questions raised by genetic redundancy, it also complicates genome engineering of $S$. cerevisiae. The conversion of substrate into product via native or engineered pathways, relies on the microbial host core pathways for the supply of metabolic precursors, energy-rich molecules and redox equivalents. These biochemical reactions are catalysed by sets of 'metabolic' genes that are characterized by a high genetic redundancy in eukaryotes (Escalera-Fanjul et al. 2019). Not only has the physiological role of many paralogous genes not been fully elucidated, but the 
manipulation of specific biochemical reactions is hindered by the presence of multiple paralogous genes with poorly known functions that are scattered over the $12 \mathrm{Mb}$, mosaic yeast genome and its 16 chromosomes. Additionally, expression of these redundant genes dissipates cellular resources (e.g., carbon, energy) that might be better invested in industrially-relevant properties such as high product yield or cellular robustness to the stressful environment of large-scale fermentation.

To tackle these fundamental and applied challenges, taking glycolysis and ethanolic fermentation as starting point, Solis-Escalante and colleagues pioneered the genetic reduction of central carbon metabolism in S. cerevisiae. The set of 26 genes encoding the (iso)enzymes catalysing 12 reactions was reduced to 13 genes (Solis-Escalante et al. 2015). Remarkably, this $50 \%$ genetic reduction did not result in any visible phenotypic effect, although a wide range of growth conditions was tested. These observations argued against gene dosage being a strong driving force in the evolution of Crabtree positive yeasts (Escalera-Fanjul et al. 2019; Solis-Escalante et al. 2015) and raised questions on the mechanisms involved the fixation of these gene duplicates in the $S$. cerevisiae genome. A recent study suggests that the role of the redundant paralogs might be highly context-dependent and that some relevant conditions were not tested by Solis-Escalante et al. (2015) (e.g. the role of Pyruvate kinase 2 in the utilization of threecarbon substrates such as dihydroxyacetone (Bradley et al. 2019)). The surprising lack of phenotype of the "Minimal Glycolysis" yeast strain (called MG) enabled the construction of a genetically simplified version of the glycolytic pathway, which was subsequently relocalized to a single chromosomal locus (Kuijpers et al. 2016). The resulting yeast strain with a single-locus glycolysis presents a powerful tool to remodel the glycolytic pathway in two single steps into any redesigned (heterologous) version.

Glycolysis is an important but small part of Central Carbon Metabolism (CCM), a set of reactions required for the conversion of carbon feedstocks into any industrially-relevant product (Fig. 1). For cells, CCM is primarily the set of reactions that convert carbon sources into the 12 building blocks required for the synthesis of cellular components (Nielsen and Keasling 2016). CCM encompasses ca. 111 genes, with $66 \%$ of duplicates (Fig. 2). Reducing its genetic complexity would be the first step in an attempt to construct a modular, designer yeast genome, with a single-locus CCM, as previously achieved for glycolysis and fermentation. Modular, specialised synthetic chromosomes could be ideal platforms for the centralisation of the CCM genes (Postma et al. 2021).

The main goal of this study was to explore the extent to which the number of genes encoding CCM enzymes in S. cerevisiae can be reduced without substantially affecting fitness under a set of chosen growth conditions. To this end, redundancies were first predicted based on literature data on gene expression, enzyme activities and phenotype of (single) deletion mutants. Subsequently, phenotypes of 
bioRxiv preprint doi: https://doi.org/10.1101/2021.08.24.457526; this version posted August 26, 2021. The copyright holder for this preprint

(which was not certified by peer review) is the author/funder, who has granted bioRxiv a license to display the preprint in perpetuity. It is made available under aCC-BY-NC-ND 4.0 International license.

mutants with mutations in sets of genes encoding CCM enzymes were tested under a wider range of growth conditions. In this first attempt of genetic reduction of yeast CCM at this scale, special attention is given to possible synergistic effects of mutations that were previously studied in separate strains. 


\section{Results}

\section{Genetic reduction strategy}

In this study the CCM of S. cerevisiae was defined as the set of biochemical reactions encompassed by glycolysis, ethanolic fermentation, pentose-phosphate pathway, acetyl-CoA synthesis, tricarboxylic acid cycle, anaplerosis, gluconeogenesis, glyoxylate cycle and glycerol metabolism. As CCM reactions occur in multiple compartments, mitochondrial transporters were also considered (Fig. 2). Transport over the peroxisomal membrane was not considered as this phenomenon is poorly studied (DeLoache et al. 2016). For the construction of a minimal CCM stain, decisions to remove or retain genes were based on (i) transcript levels from an expression compendium encompassing 170 different cultivation conditions (Knijnenburg et al. 2009), (ii) enzyme activities in cell extracts of mutant strains when data were available, and (iii) on reported phenotypes of null mutants. Genes encoding proteins with reported secondary ('moonlighting') functions or proteins known to cause auxotrophy upon deletion were retained (Gancedo and Flores 2008).

Genes were classified as functionally redundant when at least $75 \%$ of the specific growth rate of the congenic reference strains CEN.PK113-7D (Ura+) or IMX581 (Ura-) was retained during aerobic batch cultivation on synthetic medium supplied with either glucose or ethanol. Ethanol-grown cultures were included as, in contrast to glucose, ethanol can only be dissimilated by respiration and because its metabolism involves different sets of CCM enzymes and transporters. In addition, testing for growth on ethanol ensured that the intensive engineering undergone by the strains, including removal of several mitochondrial proteins, did not cause respiratory deficiency.

The previously constructed MG strain, in which 13 out of the 26 existing paralogs of genes encoding glycolytic enzymes and fermentation enzymes were deleted without the detection of major phenotypes, was used as starting point of the present CCM reduction endeavour. To identify any synergistic effects between the newly introduced deletions and the 13 deletions already present in the MG strain, a congenic naïve reference strain (IMX581) with a full complement of glycolytic and fermentation genes was also used in parallel to MG for serial deletions. To accelerate the deletion workflow, genes involved in individual pathways or processes were deleted in sets of two to four (Fig. 3). When substantial loss of fitness was observed, the contribution of individual deletions was dissected by constructing additional strains with various combinations and numbers of deletions. 


\section{Deletion of 35 CCM genes has minimal impact on specific growth rate on chemically}

\section{defined glucose medium}

\section{Pentose-phosphate pathway}

The pentose-phosphate pathway (PPP) reduces cellular $\mathrm{NADP}^{+}$, generates ribose-5-phosphate and erythrose-4-phosphate for nucleic acid and amino-acid synthesis and, in strains engineered for pentose fermentation, acts as dissimilatory pathway (Jansen et al. 2017).

Four of the seven reactions in the PPP are catalyzed by pairs of isoenzymes encoded by WGD paralogs, for which sequence similarities range from $47 \%$ (SOL3 and SOL4) to 87 \% (GND1 and GND2) (Suppl. Table S1). Based on transcript levels across a wide ranges of cultivation conditions (Knijnenburg et al. 2009), SOL4, GND2, TKL2 and NQM1 were considered as minor paralogs. Moreover, deletion of TKL2 and NQM1 was previously reported not to affect growth on glucose synthetic medium (Lobo and Maitra 1982; Matsushika et al. 2012; Schaaff-Gerstenschläger et al. 1993). While similar in vitro enzyme activities were reported for Sol3 and Sol4 in cell extracts (Stanford et al. 2004), SOL4 was deleted based on its consistently lower transcript level (Knijnenburg et al. 2009). Simultaneous deletion of GND2, TKL2, SOL4 and NQM1 in the naïve reference strain or in the MG strain, while retaining SOL3, GND1, TKL1 and TAL1, did not significantly affect growth rate on either synthetic medium with $2 \%(\mathrm{w} / \mathrm{v})$ glucose (SMD) or $2 \%$ (v/v) ethanol (SME) as carbon sources (strains IMX1592 (ppp ${ }^{\text {min }}$ ) and IMX1591 (called CCMin1: glyc ${ }^{\text {min }}$ fer $^{\text {min }}$ ppp $^{\mathrm{min}}$ ), Fig. 3). Absence of a previously reported extended lag phase and slower growth on ethanol of sol4 null mutants (Castelli et al. 2011) was not observed. This difference may be related to the use of different S. cerevisiae strain backgrounds.

\section{Tricarboxylic acid cycle, anaplerotic reactions and gluconeogenesis}

In addition to its dissimilatory role in oxidizing acetyl-CoA units to $\mathrm{CO}_{2}$, the tricarboxylic acid (TCA) cycle supplies precursors, NADH, FADH 2 and ATP (Barnett 2003). During growth on fermentable sugars, the TCA cycle is a mitochondrial pathway, with acetyl-CoA resulting from oxidative decarboxylation of pyruvate by the pyruvate dehydrogenase (PDH) complex. To replenish use of TCA-cycle intermediates for biosynthesis, the cycle's acceptor molecule oxaloacetate can be imported from the cytosol, where it is produced by carboxylation of pyruvate. The nine biochemical reactions of the TCA cycle involve 22 mitochondrial enzymes, which show little genetic redundancy. Two reactions are catalysed by single enzymes, Mdh1 and Fum1, while three steps are catalysed by complexes of two to five proteins. Deletion of genes encoding 
individual subunits of the $\alpha$-ketoglutarate and succinyl-CoA synthetase complexes renders the complexes dysfunctional (Cupp and McAlister-Henn 1991; Cupp and McAlister-Henn 1992; Dickinson et al. 1986; Przybyla-Zawislak et al. 1998; Repetto and Tzagoloff 1989; Repetto and Tzagoloff 1990). In contrast, the succinate dehydrogenase complex, in which four functions are performed by seven proteins, does show some redundancy. $S D H 1, S D H 3$ and $S D H 4$ have $S D H 1 b, S H H 3$, and $S H H 4$, respectively, as homologs originating from the WGD, while SDH2 is a unique gene (Bullis and Lemire 1994; Chapman et al. 1992; Colby et al. 1998; Gebert et al. 2011; Lombardo et al. 1990; Szeto et al. 2012). Deletion of SDH1b, SHH3 and SHH4 has minor or no effect on complex integrity and yeast physiology and these genes are considered as functionally redundant (Chang et al. 2015; Colby et al. 1998; Szeto et al. 2012). Citrate synthase (Cit1 and Cit3) and isocitrate dehydrogenase (Idh1, Idh2 and Idp1) have functionally redundant mitochondrial enzymes. Based on expression data and lack of a phenotypic difference during fermentative and respiratory growth, Cit3 and the $\mathrm{NADP}^{+}$-dependent Idp1 were considered to be redundant (Haselbeck et al. 1992; Jia et al. 1997; Knijnenburg et al. 2009; Zhao and McAlister-Henn 1996). Single deletion of ACO1 or ACO2, which encode aconitase isoenzymes, causes amino acid auxotrophies (Fazius et al. 2012; Gangloff et al. 1990). Idh1 and Idh2 are part of a complex and are both required for isocitrate dehydrogenase activity (Cupp and McAlister-Henn 1991; Cupp and McAlister-Henn 1992). Based on this information, only five of the 22 TCA cycle mitochondrial proteins were considered functionally redundant and, therefore, selected as candidates for elimination: Cit3, Idp1, Sdh1b, Shh3 and Shh4. IDP1 was targeted in a later deletion round, along with extra-mitochondrial paralogs of TCA-cycle that are part of the glyoxylate cycle.

There are several enzymes that form an interface between the TCA cycle and glycolysis. The WGD paralog pair PYC1 and PYC2 encodes isoenzymes of the anaplerotic enzyme pyruvate carboxylase. Transcript levels of these two highly similar genes (92\%, Suppl. Table S1) are condition-dependent, and despite some conflicting reports on the physiological impact of PYC1 and PYC2 deletion (Brewster et al. 1994; Knijnenburg et al. 2009; Pronk et al. 1996; Stucka et al. 1991), one study shows that only deletion of PYC1 leads to aspartate auxotrophy (Brewster et al. 1994). PYC2 was therefore deleted. Deletion of $M A E 1$, which encodes a mitochondrial malic enzyme catalyzing the oxidative decarboxylation of malate to pyruvate, does not show a clear phenotype. However, double deletion of MAE1 and PYK2 reduces the specific growth rate on ethanol by $62 \%$ (Boles et al. 1998). As PYK2 was deleted in the MG strain, MAE1 was retained. The gluconeogenic enzymes PEP carboxykinase (Pck1) and fructose-1,6-bisphosphatase (Fbp1) are essential for bypassing the irreversible pyruvate-kinase and phosphofructokinase reactions, 
respectively, during growth on non-fermentable carbon sources (Soontorngun et al. 2007; Valdés-Hevia et al. 1989).

CIT3, SDH1b, SHH3, SHH4 and PYC2 were deleted in two consecutive transformations rounds in the naïve reference strain and the CCMin1 strain ( $g l y c^{\min }$ fer $^{\min } \mathrm{ppp}^{\mathrm{min}}$ ), resulting in IMX1805 (tca ${ }^{\mathrm{min}}$ ) and IMX1806 (CCMin2: glyc ${ }^{\min }$ fer $^{\min }$ ppp $^{\min }$ tca $^{\mathrm{min}}$ ), respectively. Both strains grew as well as their parental strains in chemically defined medium supplemented with glucose or ethanol (Fig. 3).

\section{Mitochondrial Carriers}

The 35 nuclearly encoded mitochondrial carriers (MCs) mediate transport of numerous metabolites, nucleotides, cofactors and inorganic anions between mitochondrial matrix and cytosol (Palmieri et al. 2006). Based on extensive functional analysis studies (Palmieri and Monné 2016; Palmieri et al. 1996), 19 MCs involved in transport of pyruvate, TCA-cycle intermediates, CoA, ADP, ATP, Pi, NAD', FAD and thiamine pyrophosphate (cofactor of pyruvate dehydrogenase and $\alpha$-ketoglutarate dehydrogenase) were considered to be part of CCM (Suppl. Table S2, Fig. 2). Potential genetic redundancy was identified for 10 of these MCs, with protein sequence similarity varying between 51 and 87 \% (Suppl. Table S1). In addition to genetic redundancy, functional redundancy has to be considered since several genetically distinct transporters can transport the same solutes, as exemplified by the antiport of ADP and ATP across the mitochondrial membrane by three Aac homologs as well as by Sal1. Aac2 and Aac3 originate from WGD while Aac1 does not. Sal1 shares no homology with the Aac carriers and harbours an additional $\mathrm{Ca}^{2+}$ binding domain (Cavero et al. 2005; Smith and Thorsness 2008). Several studies indicate Aac2 as major paralog, whose presence suffices to sustain adenine nucleotide transport during respiratory growth (Adrian et al. 1986; Cavero et al. 2005; Chen 2004; Gawaz et al. 1990; Knijnenburg et al. 2009; Laco et al. 2010; Smith and Thorsness 2008). AAC1, AAC3 and SAL1 were therefore all candidates for deletion. NAD', synthesized in the cytosol and required for the $\mathrm{NAD}^{+}$-dependent mitochondrial dehydrogenases in $\mathrm{CCM}$, is imported by two MCs encoded by NDT1 and NDT2, paralogs with $51 \%$ similarity at the protein level (Suppl. Table S1). NDT1 and NDT2 are individually dispensable for growth on glucose or ethanol, but deletion of both precludes growth on non-fermentable carbon sources (Todisco et al. 2006). Therefore, only one of the paralogs, NDT2 was chosen for deletion. Since import of FAD, CoA and thiamine pyrophosphate is crucial for mitochondrial activity, the corresponding unique genes (FLX1, LEU5 and TPC1) were retained (Bafunno et al. 2004; Marobbio et al. 2002; Prohl et al. 2001; Tzagoloff et al. 1996). Pyruvate is located at the interface of glycolysis and the TCA cycle and, in addition, mitochondrial pyruvate is required for synthesis of branched-chain amino acids (BCAA). Pyruvate import into mitochondria is 
mediated by three isoenzymes: Mpc1, Mpc2 and Mpc3. Mpc1 is constitutively expressed and forms complexes with either of the highly homologous Mpc2 or Mpc3 isoenzymes (Bender et al. 2015). MPC2 is expressed during fermentative growth, while MPC3 is expressed during respiratory growth. Deletion of MPC2 leads to a severe growth defect, even in glucose-containing medium supplied with BCAA, while MPC3 deletion leads to a modest (20\%) decrease of specific growth rates on non-fermentable carbon sources (Herzig et al. 2012; Timón-Gómez et al. 2013). Based on these literature data, it was decided to delete MPC3.

Sfc1 and Dic1 employ different mechanisms to import succinate into mitochondria and, since both are essential for growth on ethanol (Fernández et al. 1994; Palmieri et al. 1997; Palmieri et al. 1999b), neither were eliminated. Oxaloacetate is mainly transported by Oac1, whose removal only has a minor impact on specific growth rate on glucose medium, which is linked to its secondary function as an exporter of $\alpha$-isopropylmalate for leucine biosynthesis (Marobbio et al. 2008; Palmieri et al. 1999a). Since we observed a $26 \%$ reduction of the specific growth rate on glucose upon deletion of OAC1 in the CEN.PK genetic background used in this study (Suppl. Fig. S1), it was retained in strain construction.

Four additional and partially functionally redundant MCs with different transport mechanisms and affinities mediate organic acid transport. Ctp1 is a citrate/malate antiporter, the paralogous carriers Odc1 and Odc2 are $\alpha$-ketoglutarate/oxodicarboxylate antiporters and Yhm2 exchanges $\alpha$-ketoglutarate and citrate, thereby enabling NADPH shuttling between cytosol and mitochondria (involving isocitrate dehydrogenase and aconitase) (Castegna et al. 2010; Cho et al. 1998; Kaplan et al. 1995; Kaplan et al. 1996; Palmieri et al. 2001; Scarcia et al. 2017; Tibbetts et al. 2002). Deletion of CTP1 or double deletion of $O D C 1$ and $O D C 2$ does not affect growth, while triple deletion of $Y H M 2, O D C 1$ and $O D C 2$ does (Scarcia et al. 2017; Tibbetts et al. 2002). Based on these literature data, CTP1, ODC1 and ODC2 were selected for deletion, with the realization that their combined deletion might affect di- and tri-carboxylic acids trafficking.

In total, 8 MCs were targeted for elimination. First, AAC1, AAC3, SAL1 and MPC3 were simultaneously deleted, followed by simultaneous deletion of NDT2, CTP1, ODC1 and ODC2. Deletion of AAC1, AAC3, SAL1 and MPC3 in the naïve reference strain, resulting in strain IMX2360 only marginally affected specific growth rate on SMD (3-5 \% decrease), but had a stronger impact on growth on SME (14$18 \%$ slower growth, Fig 3). These results are in agreement with the reported roles of these MCs in respiratory growth. When introduced in CCMin2 (glyc ${ }^{\min }$ fer $^{\min } \mathrm{ppp}^{\mathrm{min}}$ tca $^{\mathrm{min}}$ ), resulting in strain IMX1984, the same set of deletions did not affect specific growth rate on either SMD or SME. 
Combined deletion of NDT2, CTP1, ODC1 and ODC2 reduced specific growth rate on SMD by $23 \%$ in the naïve reference strain (resulting in strain IMX2230) and $30 \%$ in IMX1984 (glyc ${ }^{\text {min }}$ fer $^{\text {min }}$ ppp $^{\text {min }}$ tca $^{\text {min }}$ aac1 aac3 $\Delta$ sal1 $\operatorname{mpc3\Delta }$; resulting in strain IMX2231) (Fig 3). When, instead, only NDT2, CTP1 and ODC1 were deleted in the naïve reference strain (resulting in strain IMX2404) or in engineered background strain IMX1984 (resulting in strain IMX2407, called CCMin3: glyc ${ }^{\min } \mathrm{fer}^{\min } \mathrm{ppp}^{\mathrm{min}}$ tca $^{\mathrm{min}} \mathrm{mc}^{\mathrm{min}}$ ), specific growth rate on SMD was not affected and only a small (3-7 \%) reduction of growth rate was observed on SME (Suppl. Fig S2 and Fig 3). CCMin3 (glyc ${ }^{\min } \mathrm{fer}^{\min } \mathrm{ppp}^{\mathrm{min}} \mathrm{tca}^{\mathrm{min}} \mathrm{mc}^{\mathrm{min}}$ ), retained $96 \%$ of the specific growth rate of the reference strain on SMD and $82 \%$ of its specific growth rate on SME.

\section{Fumarate reductases, acetyl-CoA synthesis and glyoxylate cycle}

Cytosolic (Frds1) and mitochondrial (Osm1) fumarate reductases re-oxidise $\mathrm{FADH}_{2}$ which has been proposed to be important for protein folding under anaerobic conditions (Camarasa et al. 2007; Jouhten and Penttilä 2014; Liu et al. 2013; Neal et al. 2017). Double deletion of FRDS1 and OSM1 has no phenotypic effect on complex glucose medium under aerobic conditions (Arikawa et al. 1998). However, Osm1 has a moonlighting function outside CCM, as it contains two translation sites, leading to the targeting to the ER of an Osm1 variant. Therefore, only FRDS1 was considered for deletion in the design of a minimal CCM strain.

The glyoxylate cycle, which is essential for providing biosynthetic precursors with more than 2 carbon atoms during growth on fatty acids and two-carbon compounds, encompasses reactions in the peroxisome and cytosol (Kunze et al. 2006; Xiberras et al. 2019) and uses acetyl-CoA as substrate made by the acetyl-CoA synthesis pathway. Ethanol is converted into to acetyl-CoA via alcohol dehydrogenase (already reduced in the MG strain), acetaldehyde dehydrogenases and acetyl-CoA synthetases. Five acetaldehyde dehydrogenase isoenzymes, Ald2 to Ald6, oxidize acetaldehyde to acetate with either $\mathrm{NADP}^{+}$or NAD ${ }^{+}$as cofactor. The mitochondrial isoenzymes Ald4 and Ald5, required for growth on ethanol (Boubekeur et al. 2001; Kurita and Nishida 1999) and for maintenance of a functional respiratory chain (Kurita and Nishida 1999), were both retained. Ald6 is the major cytosolic isoenzyme, whose elimination strongly affects growth on fermentable and non-fermentable carbon sources (Meaden et al. 1997). The other two cytosolic acetaldehyde dehydrogenases, Ald2 and Ald3 are involved in conversion of 3aminopropanal to $\beta$-alanine for pantothenic acid biosynthesis (Meaden et al. 1997; White et al. 2003). As single deletion of $A L D 2$ or $A L D 3$ does affect growth on ethanol or glucose and $A L D 2$ is the major paralog in pantothenic acid production (Navarro-Aviño et al. 1999; White et al. 2003), ALD3 was considered for deletion. 
Acetate is then converted to acetyl-CoA via Acs1, whose localisation is under debate. Acs1 having been reported to occur in the cytosol, the nucleus and in peroxisomes, depending on growth conditions (Chen et al. 2012; Krivoruchko et al. 2015). Acs1 and its isoenzyme Acs2 are essential for growth on nonfermentable and fermentable carbon sources, respectively (Chen et al. 2012). The mitochondrial acetylCoA hydrolase Ach1, is also able to convert acetate into acetyl-CoA, but uses succinyl-CoA as CoA donor. Deletion of $A C H 1$ leads to reduced chronological lifespan, severe mitochondrial damage and accumulation of reactive oxygen species (Orlandi et al. 2012; Takahashi et al. 2006). ACS1, ACS2 and ACH1 were therefore retained.

The glyoxylate cycle is initiated by Cit2, an extramitochondrial isoenzyme of the mitochondrial Cit1 and Cit3 citrate synthases, whose localisation is under debate and has been reported in the cytosol and peroxisome (Chen et al. 2012; van Rossum et al. 2016a). Citrate is then converted into isocitrate in the cytosol by the dually localized enzyme Aco1 in cytosol and mitochondria (Regev-Rudzki et al. 2005). Via a series of cytosolic and peroxisomal reactions (some localisations under debate), including: the isocitrate lyase Icl1 (cytosol) (Chaves et al. 1997; Taylor et al. 1996), the malate synthase (Mls1/Mls2 cytosol and peroxisome) (Chen et al. 2012; Hiltunen et al. 2003; Kunze et al. 2002) and malate dehydrogenase (Mdh2 in cytosol and Mdh3 occurs in the peroxisome) (Hiltunen et al. 2003), the net synthesis of TCA-cycle intermediates is enabled from acetyl-CoA.

Possible redundancies of glyoxylate enzymes also involved in TCA cycle were discussed above, with only Cit3 selected for elimination in a minimal CCM strain. Since the glyoxylate cycle enzymes Cit2, Mls1, Icl1 and Mdh2 (cytosolic isoenzyme of the mitochondrial Mdh1) are either essential for growth on $\mathrm{C}_{2}$-compounds or their elimination leads to strong reductions in growth rate, they were retained in the minimal CCM design (Chen et al. 2012; Fernández et al. 1992; Hartig et al. 1992; Kim et al. 1986; Kunze et al. 2006; Lee et al. 2011; Minard and McAlister-Henn 1991). The proteins Icl2, Mls2 and Mdh3 are homologous to Icl1, Mls1 and Mdh1/Mdh2, respectively, but have (additional) functions outside CCM (Fernández et al. 1993; Hartig et al. 1992; Luttik et al. 2000) and were therefore also retained.

The peroxisomes harbour the $\mathrm{NADP}^{+}$-dependent isocitrate dehydrogenase Idp3. Deletion of its mitochondrial homologs Idp1 and Idp2 does not affect growth on ethanol or glucose (Haselbeck et al. 1992; Zhao and McAlister-Henn 1996). Idp1 and Idp2 were therefore the only genes considered for elimination in the minimal CCM design.

Triple deletion of FRDS1, IDP1 and IDP2 in the naïve reference strain (resulting in strain IMX2470: fum $^{\min }$ glyox $^{\mathrm{min}}$ ) did not affect specific growth rate on SMD and caused a $7 \%$ lower growth rate on SME (Fig.3 and Suppl. Fig. S2). Deletion in CCMin3 (IMX2407: glyc ${ }^{\min } \mathrm{fer}^{\mathrm{min}} \mathrm{ppp}^{\mathrm{min}} \mathrm{tca}^{\mathrm{min}} \mathrm{mc}^{\mathrm{min}}$ ) did not affect 
specific growth rate on either SMD or SME (Fig. 3). The resulting strain CCMin4 (IMX2475: glyc ${ }^{\min }$ fer $^{\min }$ $\mathrm{ppp}^{\min } \mathrm{tca}^{\min } \mathrm{mc}^{\mathrm{min}}$ fum $^{\min }$ glyox ${ }^{\min }$ ) retained $94 \%$ and $79 \%$ of the specific growth rate of the naïve reference strain IMX581, on SMD and SME, respectively. For reasons of experimental efficiency, ALD3 was removed in the final deletion round (see below).

\section{Glycerol synthesis}

Glycerol production is essential for redox balancing in anaerobic S. cerevisiae cultures (Van Dijken and Scheffers 1986). In addition, glycerol plays a key role in osmotolerance and maintenance of cellular volume and turgor pressure during growth under hypertonic conditions (Ansell et al. 1997; Nevoigt and Stahl 1997). The conversion of dihydroxyacetone phosphate to glycerol-3-phosphate is catalysed by the isoenzymes Gpd1 and Gpd2. gpd1 deletion mutants are osmosensitive, but show no growth defects in the absence of stress (Al-Saryi et al. 2017; Albertyn et al. 1994; Björkqvist et al. 1997). In contrast, gpd2 null mutants show a mild reduction of aerobic growth rates and strongly decreased growth rates under anaerobic conditions (Eriksson et al. 1995; Nissen et al. 2000). Glycerol-3-phosphate is converted into glycerol by the redundant Gpp1 and Gpp2 isoenzymes. Single deletion of either enzyme neither affects osmotolerance nor growth on glucose or ethanol, while gpp1 mutants have been reported to show extended lag phases in anaerobic cultures (Norbeck et al. 1996; Pahlman et al. 2001). Therefore, GPD1 and GPP2 were chosen for deletion.

Triple deletion of $A L D 3$, GPD1 and GPP2 did not significantly affect specific growth rate on SMD, while a small growth rate reduction was observed on SME in both the naive reference strain and the engineered background (IMX2509: Ace, ${ }^{\text {min }}$ Glycerol $^{\text {min }}$ and IMX2519/CCMin5: glyc ${ }^{\min }$ fer $^{\min }$ ppp $^{\text {min }}$ tca $^{\min }$ $\mathrm{mc}^{\mathrm{min}}$ fum $^{\mathrm{min}}$ glyox ${ }^{\mathrm{min}}$ Ace, ${ }^{\text {min }}$ Glycerol $^{\mathrm{min}}$, respectively Fig. 3). The lower specific growth rate on SME could be attributed to the double deletion of GPD1 and GPP2 (strain IMX2612, Fig. 3 and Suppl. Fig. S2).

The auxotrophic 35-deletion strain IMX2519 (glyc $^{\min } \mathrm{fer}^{\min } \mathrm{ppp}^{\min } \mathrm{tca}^{\mathrm{min}} \mathrm{mc}^{\mathrm{min}}$ fum $^{\min }$ glyox $^{\mathrm{min}}$ Ace, $^{\text {min }}$ Glycerol $^{\mathrm{min}}$ ) grew at $93 \%$ and $71 \%$ of the specific growth rate of control strain IMX581 on uracilsupplemented SMD and SME, respectively. Integration of a URA3 cassette yielded the uracil-prototrophic 35-deletion strain IMX2538, which was labelled 'minimal CCM strain'. This prototrophic strain grew at 94 $\%$ of the prototrophic control strain with a full set of CCM genes CEN.PK113-7D on SMD and at $76 \%$ on SME (Fig. 3). These values were within the $25 \%$ boundary that were initially set, and the physiology of the minimal CCM strain was further explored. 


\section{A S. cerevisiae strain with minimalized CCM shows only mild growth defects on synthetic}

\section{media}

The genome sequence of the minimal CCM strain was analysed by short-read and long-read techniques. Long-read sequencing revealed that 9 transformation rounds and deletion of 22 genes from the MG strain had not led to chromosomal rearrangements or deletions. Previously reported duplicated regions on chromosomes 3 and 5 of the MG strain based on karyotyping and short read sequencing (Solis-Escalante et al. 2015) were also observed in this study with long-read sequencing. Sequence analysis confirmed that all 22 targeted CCM genes were correctly deleted from the MG strain. The genome of the minimal CCM strain showed 45 Single Nucleotide Polymorphisms (SNPs) relative to the MG strain, of which eight were located in genes and only four led to an amino acid change (Table 1), none of which affected proteins involved in CCM.

The physiology of the minimal CCM strain was compared to that of the congenic reference strain CEN.PK113-7D, which has a full complement of CCM genes, in $\mathrm{pH}$-controlled aerobic bioreactor cultures on SMD. Consistent with the analyses in shake flasks, the specific growth rate of the minimal CCM strain in these cultures was $8 \%$ lower than the level of the reference strain CEN.PK113-7D (Table 2). During the glucose consumption phase, biomass-specific glucose and oxygen consumption rates of the two strains, as well as their ethanol and $\mathrm{CO}_{2}$ production rates and their biomass and ethanol yields on glucose were also similar. The minimal CCM strain did exhibit a higher acetate production rate and yield (63\% and 71 $\%$ higher, respectively) than the reference strain, a difference already observed for the MG strain (SolisEscalante et al. 2015). Similarly, a lower glycerol production rate and glycerol yield on glucose ( $27 \%$ and 23 \% lower, respectively), was in line with data reported for a gpd1 deletion mutant (Björkqvist et al. 1997). After the diauxic shift, growth of the minimal CCM strain on ethanol, glycerol and organic acids produced during the glucose phase, proceeded at a $17 \%$ lower rate than observed for the reference strain (Table 2). As a macroscopic characterization based on extracellular products might mask subtle differences of intracellular fluxes, intracellular concentrations of CCM intermediates were measured during the mid-exponential growth phase on glucose. The concentrations of these metabolites hardly differed between the minimal CCM and the control strains (Table 3). These results indicated that a $32 \%$ reduction of the complement of genes encoding CCM enzymes of S. cerevisiae had only a small impact on its physiology under standard laboratory conditions. 


\section{Dissecting individual from synergistic responses to growth on a range of conditions}

To explore genetic redundancy of CCM genes, the minimal CCM strain and the congenic reference strain CEN.PK113-7D were grown under a broad range of conditions. Some of these were chosen based on previously reported phenotypes (e.g. high osmolarity) or connection to CCM (e.g. growth on various carbon sources), while others subjected the strains to adverse conditions (e.g. acidic or alkaline $\mathrm{pH}$ ).

Consistent with reports that deletion of GPD1 causes decreased osmotolerance (Albertyn et al. 1994; Eriksson et al. 1995), the minimal CCM strain grew 13 to $25 \%$ slower than the reference strain exposed to high osmolarity, which was imposed by adding high concentrations of sorbitol (1M and $2 \mathrm{M}$ ) or glucose $(10 \%$ and $20 \% \mathrm{w} / \mathrm{v})$. Construction and analysis of strains with different combinations of deletions in reference and CCM-minimization backgrounds confirmed that this growth reduction specifically resulted from GPD1 deletion, rather than from synergistic effects (Fig. 4 and Suppl. Fig. S3). MPC3 deletion has been reported to cause lower growth rates on glycerol or lactate as sole carbon source (Timón-Gómez et al. 2013). Since Mpc3 is a pyruvate transporter, growth was assessed directly on chemically defined medium with pyruvate as sole carbon source. The minimal CCM strain grew $79 \%$ slower than the control strain, however this could surprisingly not be attributed to the MPC3 deletion (Fig. 4 and Suppl. Fig. S3).

Combined deletion of $A A C 1, A A C 3, M P C 3$ and $S A L 1$ caused a $3-5 \%$ and a $14-18 \%$ decrease of growth rates on SMD and SME, respectively, thus marking their importance on these carbon sources. According to previous reports, individual deletion of these four genes does not affect growth on glucose and individual deletion of AAC1, AAC3 and SAL1 does not affect growth on ethanol (Cavero et al. 2005; Kolarov et al. 1990; Smith and Thorsness 2008; Timón-Gómez et al. 2013). Deletion of MPC3 has been reported to cause a decrease in growth rate on glycerol and lactate (Timón-Gómez et al. 2013), and may therefore also be responsible for the lower growth rate on ethanol. Reintroduction of MPC3 in strain

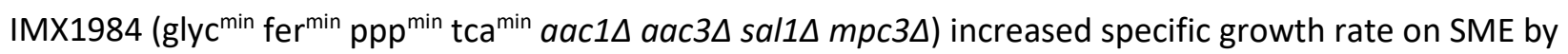
only $3 \%$, while reintroduction in IMX2519 (CCMin 5: glyc ${ }^{\min }$ fer $^{\text {min }}$ ppp $^{\text {min }}$ tca $^{\text {min }}$ mc $^{\text {min }}$ fum $^{\text {min }}$ glyox ${ }^{\text {min }}$ Ace, $^{\text {min }}$ Glycerol $^{\mathrm{min}}$ ) did not affect growth rate (Suppl. Fig. S3). These results suggest that the observed impact of quadruple deletion of $A A C 1, A A C 3, M P C 3$ and $S A L 1$ on ethanol growth was caused by synergistic effects.

Some paralogs have been reported to be specifically important under anaerobic conditions (AAC3, FRDS1) (Arikawa et al. 1998; Kolarov et al. 1990). In line with these reports, while the MG strain showed the same growth rate as CEN.PK113-7D under anaerobic conditions, the minimal CCM strain showed a 25 $\%$ lower anaerobic growth rate (Fig. 4). However, testing of deletions in the reference background indicated that this difference was not caused by the deletions of AAC3 or FRDS1 (Suppl. Fig. S3). 
bioRxiv preprint doi: https://doi.org/10.1101/2021.08.24.457526; this version posted August 26, 2021. The copyright holder for this preprint

(which was not certified by peer review) is the author/funder, who has granted bioRxiv a license to display the preprint in perpetuity. It is made available under aCC-BY-NC-ND 4.0 International license.

Over the broad range of conditions tested, including several stress conditions, few differences in specific growth rate were observed (Fig. 4). Combining the phenotype of strains with individual and clustered deletions enabled to identify synergistic interactions between minor paralogs. 


\section{Discussion}

Genetic reduction has been applied in several microorganisms (Lara and Gosset 2019), including B. subtilis (Morimoto et al. 2008), E.coli (Hashimoto et al. 2005), Lactococcus lactis (Zhu et al. 2017), C. glutamicum (Suzuki et al. 2005), Streptomyces species (Komatsu et al. 2010), Pseudomonas species (Lieder et al. 2015) and Schizosaccharomyces pombe (Sasaki et al. 2013), with the purpose of discovering a minimal genome content and/or for engineering efficient cell factories. In S. cerevisiae, Marakami et al. reduced genome content by $5 \%$ by deleting 15 terminal chromosomal regions (Murakami et al. 2007). Moreover, the creation of a synthetic yeast genome in the Sc 2.0 project is accompanied by an $8 \%$ genome reduction by deletion of long terminal repeats, retrotransposons and introns; and engineering of a single chromosome yeast strain was characterised by a $9 \%$ decrease in DNA (Richardson et al. 2017; Shao et al. 2018). Genome reduction studies typically target two types of DNA sequences: non-expressed DNA (cryptic genes, mobile DNA) and irrelevant/non-essential genes. These DNA elements can be targeted by random strategies for which little knowledge is required, such as transposon mutagenesis or the elegant SCRaMbLE technique used for the recent reduction of left synthetic chromosome arm XII in S. cerevisiae (Luo et al. 2021). In the present study, knowledge-based reduction of the gene complement for CCM in S. cerevisiae was informed by gene expression data and previous phenotypic analysis on single-knockout mutants (Luo et al. 2021; Murakami et al. 2007; Richardson et al. 2017; Shao et al. 2018).

In this study, we reduced genetic complexity of central carbon metabolism (CCM) in S. cerevisiae by deletion of 35 genes encoding enzymes and transporters. This reduction corresponded to elimination of $32 \%$ of the (iso)enzymes and transporters involved in the included processes, without major impacts on strain physiology, which was tested under a broad range of conditions (Fig. 2-4). The present study built on earlier work by Solis-Escalante et al. (2015), who eliminated $50 \%$ of isoenzymes involved in glycolysis and ethanolic fermentation with a similar small impact on physiology. The attainable reduction of gene sets for enzymes and transporters involved in other CCM pathways differed, with $50 \%$ for fumarate reduction and glycerol synthesis, $37 \%$ for the mitochondrial carriers, $36 \%$ for the pentosephosphate pathway, $23 \%$ for the TCA cycle, $14 \%$ for the glyoxylate cycle, $20 \%$ for the glycolysis - TCA cycle interface plus gluconeogenesis, and $8 \%$ for acetyl-CoA metabolism. The lower attainable genetic reduction of the four latter pathways can be largely attributed to neofunctionalization and relocalization of enzymes during evolution.

Our results show that yeast CCM is remarkably robust to genetic reduction, in particular during growth on glucose, yeast's favourite carbon source, but also when challenged by a broad range of growth conditions. Notable exceptions were growth on pyruvate (79\% growth rate reduction), anaerobic growth 
on glucose ( $25 \%$ reduction), growth on ethanol ( $24 \%$ reduction) and growth at high osmolarity (between $13 \%$ and $25 \%$ lower specific growth rates). Growth-rate reductions on ethanol and at high osmolarity could be attributed to specific genes or gene combinations, while for growth on pyruvate and anaerobic growth some hypothetical targets could be excluded. The physiological role of most deleted paralogs remains elusive. Such a situation is exemplified by TKL2 and NQM1, which are paralogs of the major PPP genes TKL1 and TAL1, respectively. In S. cerevisiae strains engineered for L-arabinose utilisation, their deletion was shown to lead to lower growth rates on this pentose sugar (Matsushika et al. 2012; Wisselink et al. 2010). Clearly, as pentoses are not natural carbon sources for S. cerevisiae, this role cannot have provided an evolutionary driving force for fixation of these paralogs in its genome, but it does indicate potential contribution to fitness under other, as yet unidentified growth conditions. Testing the minimal CCM strain under an even wider variety of environmental conditions, including dynamics in nutrient availability and other environmental parameters may reveal physiological roles of these and other paralogs. Alternatively, the mechanisms that fixed some paralogs during evolution may have been disrupted by relatively recent mutations or gene loss (Shen et al. 2018). Following this reasoning, absence of a clear phenotype of knock-out mutants may have captured a stage in the evolutionary trajectory of $S$. cerevisiae that will eventually lead to loss of a paralog, evolution towards complete sub-functionalisation, or retention of functional overlap with asymmetric divergence (Kuzmin et al. 2020).

In this first step towards the genetic minimization of CCM in yeast, choices had to be made on which pathways and genes were considered as part of the CCM and on criteria for redundancy. For instance, transport of $\mathrm{NAD}^{+}, \mathrm{FAD}^{+}, \mathrm{ADP} / \mathrm{ATP}$ and Pi across the mitochondrial membrane was considered, while transport of $\mathrm{NAD}(\mathrm{P}) \mathrm{H}$, which requires more complex shuttle systems (Bakker et al. 2001; Miyagi et al. 2009) was not yet included. In addition, as S. cerevisiae cannot synthetize carnitine (Swiegers et al. 2001), the carnitine shuttle system transporting acetyl-CoA across compartments was not considered. Since CRC1, CAT1, YAT1 and YAT2 involved in this shuttle are dispensable for growth in the absence of carnitine (Swiegers et al. 2001), they can be considered for further genetic reduction of the CCM. Some genes required for anaerobic growth, such as $A D H 3$ (Bakker et al. 2000; Bakker et al. 2001) were also retained, but could be removed if fast anaerobic growth is excluded as a criterion. Several other processes and pathways are of particular interest for development of strain platforms for modular engineering yeast CCM. In this context, glucose uptake, which involves a set of 20 hexose transporters (Wieczorke et al. 1999) provides and interesting target for future experiments, whose minimization can benefit from a recently constructed $\mathrm{Hxt}^{0}$ CRISPR kit (Wijsman et al. 2019). Another logical target for minimization is 
uptake and assimilation of (alternative) carbon sources and especially of maltose, whose metabolism is enabled by highly redundant subtelomeric genes (Brickwedde et al. 2018; Brown et al. 2010).

Genetic reduction presents a first, indispensable step towards the construction of modular yeast strains for extensive remodelling of CCM. Current demands for economically competitive cell factories, with optimized titer, rate and yield (TRY), requires extensive remodelling of the CCM for the supply of precursors, (redox) cofactors and energy-rich molecules (Aslan et al. 2017; François et al. 2020; Papagianni 2012). For instance, the extensive remodelling of the native Entner-Doudoroff (ED) glycolytic pathway into the Embden-Meyerhof-Parnas (EMP) pathway improved carotenoid synthesis in Pseudomonas putida (Sánchez-Pascuala et al. 2019). Similarly, substantial efforts are invested in remodelling yeast CCM in S. cerevisiae to increase the supply of cytosolic acetyl-CoA, a precursor for a wide array of attractive biomolecules (Fig. 1) (van Rossum et al. 2016b). Also, production of complicated chemical structures like plant natural products in S. cerevisiae requires extensive remodelling of the entire central carbon metabolism (Koopman et al. 2012; Liu et al. 2019; Meadows et al. 2016). As demonstrated by Kuijpers et al. (2016), genetic reduction facilitates the colocalization of sets of genes in 'pathway clusters' and strongly accelerates the genetic remodelling of these pathways. With this strategy, the 12 steps of glycolysis and ethanolic fermentation were rapidly and efficiently swapped with heterologous variants and enabled the implementation of an innocuous DNA and RNA watermarking method (Boonekamp et al. 2020). A similar strategy can be considered for remodelling CCM, with the minimal CCM strain as starting point. As recently demonstrated, 44 transcriptional-unit sized DNA fragments can be assembled in $S$. cerevisiae into specialized, synthetic supernumerary chromosomes (Postma et al. 2021). Since the capacity of HR was not reached, assembly of synthetic chromosomes containing the set of 76 genes encoding the minimal CCM has now become a realistic objective. Subsequent CRISPR-Cas assisted removal of the duplicate CCM genes from their native locations could then generate powerful platforms for chromosome swapping and combinatorial CCM remodelling studies. The reduction of genetic complexity demonstrated in the present study therefore not only provides new insights in genetic redundancy of CCM, but also contributes to the eventual localization of all genes required for a minimized CCM on specialized, synthetic supernumerary chromosomes that allow for extensive, combinatorial remodelling of yeast metabolism for industrial applications. 


\section{Materials and Methods}

\section{Strains, media and maintenance}

The Saccharomyces cerevisiae strains used in this study are all derived from the CEN.PK family (Entian and Kötter 2007; Nijkamp et al. 2012a) (Suppl. Table S3). The naïve, uracil auxotrophic and Cas9 containing strain, IMX581 (Mans et al. 2015) and the uracil auxotrophic MG (minimal glycolysis) strain IMX370 (SolisEscalante et al. 2015) were used for deletion of genes encoding enzymes or transporters involved in central carbon metabolism (CCM). The naïve uracil prototrophic strain CEN.PK113-7D was used for physiological comparison. Complex medium used for propagation of yeast strains consisted of $10 \mathrm{~g} \mathrm{~L}^{-1}$ Bacto yeast extract, $20 \mathrm{~g} \mathrm{~L}^{-1}$ Bacto peptone and $20 \mathrm{~g} \mathrm{~L}^{-1}$ glucose (YPD), autoclaved at $110^{\circ} \mathrm{C}$ for $20 \mathrm{~min}$. After transformation, yeast strains were selected in synthetic medium (SM) (Verduyn et al. 1992) containing $3.0 \mathrm{~g} \mathrm{~L}^{-1} \mathrm{KH}_{2} \mathrm{PO}_{4}, 0.5 \mathrm{~g} \mathrm{~L}^{-1} \mathrm{MgSO}_{4} \cdot 7 \mathrm{H}_{2} \mathrm{O}, 5.0 \mathrm{~g} \mathrm{~L}^{-1}\left(\mathrm{NH}_{4}\right)_{2} \mathrm{SO}_{4}$ and $1.0 \mathrm{~mL} \mathrm{~L}^{-1}$ trace elements autoclaved at $121^{\circ} \mathrm{C}$ for $20 \mathrm{~min}$, whereafter $1.0 \mathrm{~mL} \mathrm{~L}^{-1}$ of filter sterilized vitamin solution was added. Before autoclaving media were set to $\mathrm{pH} 6$ by $1 \mathrm{M} \mathrm{KOH}$ addition. SM was supplemented with $20 \mathrm{~g} \mathrm{~L}^{-1}$ glucose (SMD) or $2 \%$ ethanol v/v (SME) for propagation and growth characterisation. Synthetic medium was supplemented with $150 \mathrm{mg} \mathrm{L}^{-1}$ uracil for uracil auxotrophic strains. For selection of transformants carrying the amdS selection marker (Solis-Escalante et al. 2013), ammonium sulfate was replaced as nitrogen source with $10 \mathrm{mM}$ of acetamide. For experiments on SM in the growth profiler and under anaerobic conditions, ammonium sulfate was replaced by $2.3 \mathrm{~g} \mathrm{~L}^{-1}$ Urea. For both media in which ammonium sulfate was replaced, $6.6 \mathrm{~g} \mathrm{~L}^{-1} \mathrm{~K}_{2} \mathrm{SO}_{4}$ was added. Growth was performed in $500 \mathrm{~mL}$ shake flask containing $100 \mathrm{~mL}$ medium or in $100 \mathrm{~mL}$ shake flasks containing $20 \mathrm{~mL}$ medium at $30{ }^{\circ} \mathrm{C}$ and $200 \mathrm{rpm}$ in an Innova 44 Incubator shaker (New Brunswick Scientific, Edisan, NJ). Culture on solid media were incubated for 3-5 days at $30^{\circ} \mathrm{C}$.

CEN.PK113-7D, IMX372, IMX2538 and several intermediate strains were tested in the growth profiler on a variety of liquid media, containing: SM(urea) plus $2 \%$ glucose (SMD), SMD at pH 3.0, 3.5 or 7.5, SM plus $10 \%$ glucose (SMD (10\%)), SM plus $20 \%$ glucose (SMD (20\%)), SMD plus 1 or 2M sorbitol, SMD plus 200 or $500 \mathrm{mM} \mathrm{NaCl}$, SM plus $111 \mathrm{mM}$ galactose (SMGal), SM plus $55 \mathrm{mM}$ maltose (SMMal), SM plus 55mM sucrose (SMSuc), SM plus $111 \mathrm{mM}$ fructose (SMFruc), SM plus $125 \mathrm{mM}$ acetic acid, SMD plus $1 \mathrm{mM}$ paraquat and YPD ( 2 \% glucose). Growth on SM plus $83.3 \mathrm{mM}$ pyruvic acid was performed in shake flasks. For anaerobic growth in shake flasks SMD was supplemented with $0.01 \mathrm{~g} \mathrm{~L}^{-1}$ ergosterol and $0.42 \mathrm{~g}$ $\mathrm{L}^{-1}$ Tween 80 dissolved in ethanol (SMD (2 \%) anaerobic) (Verduyn et al. 1992). 
Plasmids were propagated in and isolated from chemically competent Escherichia coli XL1-Blue cells, which were cultivated in Lysogeny Broth containing $10 \mathrm{~g} \mathrm{~L}^{-1}$ Bacto tryptone, $5.0 \mathrm{~g} \mathrm{~L}^{-1}$ Bacto yeast extract and $5 \mathrm{~g} \mathrm{~L}^{-1} \mathrm{NaCl}$ supplemented with $100 \mathrm{mg} \mathrm{L}^{-1}$ ampicillin (LB-amp) when required. E. coli was cultivated in $15 \mathrm{~mL}$ Greiner tubes containing $5 \mathrm{~mL}$ medium at $37{ }^{\circ} \mathrm{C}$ and $200 \mathrm{rpm}$ in an Innova 4000 Incubator shaker (New Brunswick Scientific). Bacterial cultures on solid medium were incubated overnight at $37^{\circ} \mathrm{C}$.

For solid medium $20 \mathrm{~g} \mathrm{~L}^{-1}$ of agar was added before autoclaving. All S. cerevisiae and E.coli strains were stored at $-80^{\circ} \mathrm{C}$ in $1 \mathrm{ml}$ aliquots containing $30 \% \mathrm{v} / \mathrm{v}$ glycerol in appropriate medium.

\section{Molecular biology techniques}

Plasmids were isolated from E. coli using the GenElute Plasmid Miniprep Kit (Sigma-Aldrich, St. Louis, MO) or the GeneJET Plasmid Miniprep Kit (Thermo Fisher Scientific, Waltham, MA) according to the provided protocols. DNA fragments for plasmid construction or integrative DNA fragments used in yeast transformation were amplified using Phusion High-Fidelity DNA Polymerase (Thermo Fisher Scientific) according to manufacturer's instructions, using PAGE-purified or desalted oligonucleotides (SigmaAldrich) depending on the application. Purification of genomic Polymerase Chain Reaction (PCR) amplified DNA was performed with the GenElute PCR Clean-Up kit (Sigma-Aldrich) or the GeneJET PCR Purification Kit (Thermo Fisher Scientific) if no a-specific products were present. When a-specific products were present or when DNA was amplified from plasmids, the DNA was purified by separation using electrophoresis on a $1 \%(\mathrm{w} / \mathrm{v})$ agarose gel (TopVision Agarose, Thermo Fisher Scientific) in 1x Tris-acetateEDTA (TAE) buffer (Thermo Fisher Scientific) or on a $2 \%(w / v)$ agarose gel (TopVision Agarose, Thermo Fisher Scientific) in 1x Tris-Borate-EDTA (TBE) buffer (Thermo Fisher Scientific) with subsequent purification with the Zymoclean Gel DNA Recovery kit (Zymo Research). Chemical transformation of E. coli XL1-Blue was performed by thawing of competent cells on ice, addition of DNA, followed by a heat shock for $40 \mathrm{~s}$ at $42^{\circ} \mathrm{C}$. Subsequently, cells were incubated on ice for 2 minutes and plated immediately on selective LB-amp plates and grown overnight at $37^{\circ} \mathrm{C}$. Transformation of S. cerevisiae was performed using the lithium acetate/single-stranded carrier DNA/polyethylene glycol method (Gietz and Woods 2002). Single-cell lines were obtained by three consecutive re-streaks on selective solid medium. Yeast genomic DNA was extracted according to Looke et al. (2011), the YeaStar Genomic DNA kit (Zymo Research, Irvine, CA) according to Protocol I supplied by the manufacturer or the QIAGEN Blood \& Cell Culture Kit with 100/G or 20/G Genomic-tips (Qiagen, Hilden, Germany) following the manufacturer's recommendations. Verification of the accurate genotype of engineered S. cerevisiae strains and E. coli plasmids was done by 
diagnostic PCR before strain storage at $-80^{\circ} \mathrm{C}$. These diagnostic PCRs were performed using desalted oligonucleotides and the DreamTaq PCR master Mix (Thermo Fisher Scientific) according to the manufacturer's protocol.

\section{Plasmid and strain construction}

Deletions were performed using CRISPR/Cas9. CRISPR/Cas9-based genome editing of S. cerevisiae was performed as described by Mans et al.(2015) with minor alterations. Plasmids containing a single gRNA (Suppl. Table S4) were constructed via Gibson assembly with a backbone containing the marker cassette and one insert fragment containing the gRNA and the $2 \mu \mathrm{m}$ fragment. The backbone was amplified from a pMEL plasmid (Mans et al. 2015) with primers 5980 and 5792 and the insert fragment was amplified with a gRNA specific primer designed with the yeastriction tool (Mans et al. 2015) and primer 5979 (primers in Suppl. Table S5). Plasmids containing two gRNAs were constructed using one backbone fragment and two insert fragments, each containing one gRNA and one half of the $2 \mu \mathrm{m}$ fragment. Backbones were PCR amplified from the pROS plasmids (Mans et al. 2015) with the double-binding primer 6005. Insert fragments were obtained with the gRNA specific primers together with either primer 5974 or primer 5975 (Primers in Suppl. Table S5). The backbone and gRNA insert fragment(s) were gel purified, Dpnl digested (Thermo Fisher Scientific) and Gibson-assembled in a final volume of $5 \mu l$ using NEBuilder HiFi DNA assembly master mix (NEB, Ipswich, MA), according to manufacturer's instructions. Assembled plasmids were transformed and subsequently isolated from LB-amp grown E. coli. Correct assembly was checked using diagnostic PCR (Suppl. Table S6).

IMK588 was constructed by integrating the KanMX marker at the OAC1 locus of CEN.PK113-7D. The KanMX marker with homologous flanks to OAC1 was amplified with primers 6358 and 6359 from pUG6 (Suppl. Table S4, S7 and S8).

In order to perform CRISPR editing in the MG strain (IMX370), Cas9 was integrated by transforming a Cas 9 and natNT2 DNA fragment, which can assemble by homologous recombination at the CAN1 locus. The Cas9 fragment (can1 flank-Cas9 expression cassette-SHR A) was PCR amplified with primers 2873 and 4653 from plasmid p414-TEF1p-Cas9-CYC1t (Suppl. Table S4 and S9). The natNT2 fragment (SHR A-NatNT2 marker cassette-can 1 flank) was amplified with primers 3093 and 5542 from plasmid pUG-natNT2 (Suppl. Table S4 and S9). The Cas9 containing MG strain was stocked as IMX1331.

For genome editing using CRISPR, S. cerevisiae strains were transformed with $1 \mu \mathrm{g}$ of each gRNA plasmid and $1 \mu \mathrm{g}$ of each 120 bp double-stranded DNA repair fragment. These repair fragments were made by annealing of complimentary oligonucleotides listed in Suppl. Table S7, and consisted 60 bp 
homology sequences immediately upstream of the start codon and downstream of the stop codon of the targeted gene, unless stated otherwise. Transformants were plated on selective medium. Gene deletion was verified by diagnostic colony PCR on randomly picked colonies by using the primers which bind outside of the targeted open reading frame (Suppl. Table S8). gRNA plasmids were removed by growing the colonies in liquid YPD medium and subsequent plating on solid YPD medium. Plasmid removal was confirmed by growth on selective and non-selective solid media after which the strains were stored. For all transformations the corresponding gRNA plasmids and repair fragment are summarised in Suppl. Table S10.

To obtain a prototrophic strain with 35 deletions, a URA3 transcriptional unit amplified from CEN.PK113-7D DNA (primers: 17752 and 17753, Suppl. Table S7) was integrated at the GPP2 locus of strain IMX2520 (34 deletions). The flanks of the URA3 repair fragment were homologous to the $60 \mathrm{bp}$ immediately upstream and downstream of the GPP2 ORF. The prototrophic 35 deletion strain IMX2538 was checked by diagnostic PCR, short-read sequencing and long-read nanopore sequencing. Integration of the MPC3 transcriptional unit at the X2 locus of IMX1984 and IMX2519 was achieved by amplifying the respective fragment from CEN.PK113-7D genomic DNA (primers:18025 and 18026 Suppl. Table S7) and integration by CRISPR/Cas9 using gRNA plasmid pUDR376, resulting in strains IMX2640 and IMX2641 respectively. Correct integration was verified by diagnostic PCR (Suppl. Table S8).

\section{Sequencing}

High-quality genomic DNA of yeast for sequencing was extracted using the QIAGEN Blood \& Cell Culture Kit with 100/G or 20/G Genomic-tips (QIAGEN) according to the manufacturer's instructions. DNA concentration was measured using the BR ds DNA kit (Invitrogen, Carlsbad, CA) and a Qubit ${ }^{\circledR} 2.0$ Fluorometer (Thermo Fisher Scientific). The purity was verified with a Nanodrop 2000 UV-Vis Spectrophotometer (Thermo Fisher Scientific).

\section{Short read sequencing}

IMX2538 (35 deletions prototrophic strain) was sequenced using 300 bp paired-end sequencing reads prepared with the MiSeq Reagent Kit v3 on an Illumina MiSeq sequencer (Illumina, San Diego, CA). To this end, extracted DNA was mechanically sheared to $550 \mathrm{bp}$ with the M220 ultrasonicator (Covaris, Wolburn, MA) and subsequently, the TruSeq DNA PCR-Free Library Preparation kit (Illumina) was employed to make a six-strain library. The samples were quantified by qPCR on a Rotor-Gene Q PCR cycler (Qiagen) using the KAPA Library Quantification Kit (Kapa Biosystems, Wilmington, MA). Library integrity and fragment size 
were determined with a Tapestation 2200 (Agilent Technologies). Sequencing reads were mapped onto the CEN.PK113-7D (Salazar et al. 2017) reference genome using the Burrows-Wheeler Alignment (BWA) tool (version 0.7.15) (Li and Durbin 2009) and further processed using SAMtools (version 1.3.1) (Li et al. 2009) and Pilon (with -vcf setting; version 1.18) (Walker et al. 2014) to identify single nucleotide polymorphisms (SNPs). The sequence was analysed by visualising the generated bam files in the Integrative Genomics Viewer (IGV) software (version 2.4.0) (Thorvaldsdottir et al. 2013). Chromosomal copy number was estimated by the Magnolya algorithm (version 0.15) (Nijkamp et al. 2012b).

\section{Long read sequencing}

High quality DNA of IMX2538 was isolated and checked on quantity and quality as described above. Furthermore, quality and integrity of DNA was checked with a TapeStation 2200 (Agilent Technologies, Santa Clara, CA). IMX2538 was sequenced in-house on a single R10 flow cell (FLO-MIN111) using the SQK-LSK109 sequencing kit (Oxford Nanopore Technologies, Oxford, United Kingdom), according to the manufacturer's instructions. With MinKnow (version 3.6.5, Oxford Nanopore Technologies) raw signal files were generated. Basecalling was performed by Guppy (version 4.0.11, Oxford Nanopore Technologies), followed by de novo assembly with Canu (version 2.0 (Koren et al. 2017)).

Short- and long-read sequencing data are available at NCBI under BioProject PRJNA757356.

\section{Growth rate measurement in shake flasks}

The growth rate of the constructed strains was determined in $500 \mathrm{~mL}$ shake flasks containing $100 \mathrm{~mL}$ of SMD or SME medium. Wake-up cultures were inoculated with a $1 \mathrm{~mL}$ aliquot of a strain stored at $-80{ }^{\circ} \mathrm{C}$ and grown until late-exponential phase. Pre-cultures were inoculated from the wake-up cultures and grown to mid-exponential phase. Finally, measuring cultures were inoculated in biological duplicate from the pre-culture at an initial $\mathrm{OD}_{660}$ of 0.3 . Cultures were monitored for $\mathrm{OD}_{660}$ with a Jenway 7200 spectrophotometer in technical duplicate (Cole-Parmer, Vernon Hills, IL). A maximum specific growth rate $\left(\mu_{\max }\right)$ was calculated from at least five data points in the exponential phase with at least 2 doublings.

Anaerobic shake flask-based experiments were performed at $30{ }^{\circ} \mathrm{C}$ in a Bactron anaerobic chamber (Sheldon Manufacturing Inc., Cornelius, OR) with an atmosphere of $5 \%(\mathrm{v} / \mathrm{v}) \mathrm{H}_{2}, 6 \%(\mathrm{v} / \mathrm{v}) \mathrm{CO}_{2}$ and $89 \%$ (v/v) $\mathrm{N}_{2}$, on a IKA KS 260 basic shaker at $200 \mathrm{rpm}$, using $50 \mathrm{~mL}$ shake flasks containing $30 \mathrm{~mL}$ SMD (2 \%) anaerobic medium. 


\section{Growth rate measurement in microtiter plates}

Growth measurements of strains in microtiter plate with a Growth Profiler 960 (EnzyScreen BV, Heemstede, The Netherlands) were performed as described by Postma et al. (2021).

\section{Physiological characterisation of CEN.PK113-7D and IMX2538 in bioreactor cultures}

Aerobic batch bioreactor cultures were performed in 2-L bioreactors (Applikon, Delft, The Netherlands). Bioreactors were filled with synthetic medium containing $5.0 \mathrm{~g} \mathrm{~L}^{-1}\left(\mathrm{NH}_{4}\right)_{2} \mathrm{SO}_{4}, 3.0 \mathrm{~g} \mathrm{~L}^{-1} \mathrm{KH}_{2} \mathrm{PO}_{4}, 0.5 \mathrm{~g} \mathrm{~L}^{-1}$ $\mathrm{MgSO}_{4} \cdot 7 \mathrm{H}_{2} \mathrm{O}$, and $1.0 \mathrm{~mL} \mathrm{~L}^{-1}$ trace elements. After heat sterilisation, $20 \mathrm{~g} \mathrm{~L}^{-1}$ glucose, $0.2 \mathrm{~g} \mathrm{~L}^{-1}$ antifoam emulsion C (Sigma-Aldrich, St. Louis, MA), and filter-sterilised vitamins were added to complete the medium. Upon inoculation, bioreactors contained a working volume $1.4 \mathrm{~L}$ and the culture $\mathrm{pH}$ was maintained at 5.0 by automated addition of $2 \mathrm{M} \mathrm{H}_{2} \mathrm{SO}_{4}$ or $2 \mathrm{M} \mathrm{KOH}$. Temperature was kept stable at $30^{\circ} \mathrm{C}$ and mixing of the medium was performed at $800 \mathrm{rpm}$. The gas flow was set to $700 \mathrm{~mL}$ of air per minute to supply oxygen and remove produced carbon dioxide, and an overpressure of 0.3 bar was applied to the reactor. Dissolved oxygen tension was thus maintained for all reactors above $59 \%$ over the whole duration of the batch cultivation. Off-gas was cooled to $2^{\circ} \mathrm{C}$ in a condenser on the bioreactor to prevent water evaporation, and further dried with a Permapure MD-110-48P-4 filter dryer (Permapure, Lakewood, NJ) for subsequent analysis of carbon dioxide and oxygen percentages by a MultiExact 4100 gas analyser (Servomex, Zoetermeer, The Netherlands). For both CEN.PK113-7D and IMX2538 reactors were run in biological triplicate and inoculated from exponentially growing shake flask cultures.

Optical densities were measured in technical triplicates on Jenway 7200 spectrophotometer (ColeParmer) at $660 \mathrm{~nm}$, while cell dry weights were determined by filtration of $10 \mathrm{~mL}$ of well-mixed sample over dried PES membrane filters with a pore size of $0.45 \mu \mathrm{m}$ (Pall Corporation, Port Washington, NY). Filters were washed with demineralized water and dried in a microwave oven for 20 minutes at $360 \mathrm{~W}$. Extracellular organic acids, sugars and ethanol were determined by high performance liquid chromatography (HPLC) analysis using an Aminex HPX-87H ion-exchange column (Agilent, Santa Clara) with $5 \mathrm{mM} \mathrm{H}_{2} \mathrm{SO}_{4}$ as mobile phase and a flow rate of $0.6 \mathrm{~mL} \mathrm{~min}{ }^{-1}$ at $60^{\circ} \mathrm{C}$. Glucose, glycerol, and ethanol were detected by a refractive-index detector (Agilent G1362A) and organic acids by a dual-wavelength absorbance detector (Agilent G1314F).

During mid-exponential growth in the glucose consumption phase, intracellular metabolite samples were taken with a filtration-based washing method according to Douma et al. (2010) with some modifications. Briefly, approximately $3 \mathrm{~mL}$ of cell culture was sampled in $15 \mathrm{~mL}$ of $100 \%$ methanol at $-40^{\circ} \mathrm{C}$. Biomass was washed with cooled $100 \%$ methanol on a PES membrane with a pore size of $0.45 \mu \mathrm{m}$ (Pall Corporation) 
which was pre-cooled and wetted with $100 \%$ methanol at $-40^{\circ} \mathrm{C}$. Finally, metabolites were extracted with $75 \%$ of boiling ethanol. $100 \mu \mathrm{L}$ of ${ }^{13} \mathrm{C}$ cell extract was added to each tube as an internal standard for metabolite quantification (Wu et al. 2005). The intracellular CCM metabolites, cofactors and nucleotides were derivatized and quantified as described by de Jonge et al. (2011) and Niedenführ et al. (2016). 


\section{References}

Acharya D, Ghosh TC. Global analysis of human duplicated genes reveals the relative importance of whole-genome duplicates originated in the early vertebrate evolution. BMC genomics 2016;17: 1-14.

Adrian GS, McCammon MT, Montgomery DL et al. Sequences required for delivery and localization of the ADP/ATP translocator to the mitochondrial inner membrane. Mol Cell Biol 1986;6: 626-34.

Al-Saryi NA, Al-Hejjaj MY, van Roermund CWT et al. Two NAD-linked redox shuttles maintain the peroxisomal redox balance in Saccharomyces cerevisiae. Sci Rep 2017;7: 11868.

Albertyn J, Hohmann S, Thevelein JM et al. GPD1, which encodes glycerol-3-phosphate dehydrogenase, is essential for growth under osmotic stress in Saccharomyces cerevisiae, and its expression is regulated by the high-osmolarity glycerol response pathway. Mol Cell Biol 1994;14: 4135-44.

Ansell R, Granath K, Hohmann S et al. The two isoenzymes for yeast NAD+-dependent glycerol 3phosphate dehydrogenase encoded by GPD1 and GPD2 have distinct roles in osmoadaptation and redox regulation. Embo j 1997;16: 2179-87.

Arikawa $\mathrm{Y}$, Enomoto $\mathrm{K}$, Muratsubaki $\mathrm{H}$ et al. Soluble fumarate reductase isoenzymes from Saccharomyces cerevisiae are required for anaerobic growth. FEMS Microbiol Lett 1998;165: 111-6.

Aslan S, Noor E, Bar-Even A. Holistic bioengineering: rewiring central metabolism for enhanced bioproduction. Biochem J 2017;474: 3935-50.

Bafunno V, Giancaspero TA, Brizio C et al. Riboflavin uptake and FAD synthesis in Saccharomyces cerevisiae mitochondria: involvement of the Flx1p carrier in FAD export. J Biol Chem 2004;279: 95-102.

Bakker BM, Bro C, Kötter P et al. The mitochondrial alcohol dehydrogenase Adh3p is involved in a redox shuttle in Saccharomyces cerevisiae. J Bacteriol 2000;182: 4730-7.

Bakker BM, Overkamp KM, van Maris AJ et al. Stoichiometry and compartmentation of NADH metabolism in Saccharomyces cerevisiae. FEMS Microbiol Rev 2001;25: 15-37.

Barnett JA. A history of research on yeasts 6: the main respiratory pathway. Yeast 2003;20: 1015-44.

Bender T, Pena G, Martinou JC. Regulation of mitochondrial pyruvate uptake by alternative pyruvate carrier complexes. Embo j 2015;34: 911-24.

Björkqvist S, Ansell R, Adler L et al. Physiological response to anaerobicity of glycerol-3-phosphate dehydrogenase mutants of Saccharomyces cerevisiae. Appl Environ Microbiol 1997;63: 128-32.

Boles E, de Jong-Gubbels P, Pronk JT. Identification and characterization of MAE1, the Saccharomyces cerevisiae structural gene encoding mitochondrial malic enzyme. J Bacteriol 1998;180: 2875-82.

Boonekamp FJ, Dashko S, Duiker D et al. Design and Experimental Evaluation of a Minimal, Innocuous Watermarking Strategy to Distinguish Near-Identical DNA and RNA Sequences. ACS Synth Biol 2020;9: 1361-75.

Boubekeur S, Camougrand N, Bunoust O et al. Participation of acetaldehyde dehydrogenases in ethanol and pyruvate metabolism of the yeast Saccharomyces cerevisiae. Eur J Biochem 2001;268: 505765.

Bradley PH, Gibney PA, Botstein D et al. Minor Isozymes Tailor Yeast Metabolism to Carbon Availability. mSystems 2019;4.

Brewster NK, Val DL, Walker ME et al. Regulation of pyruvate carboxylase isozyme (PYC1, PYC2) gene expression in Saccharomyces cerevisiae during fermentative and nonfermentative growth. Arch Biochem Biophys 1994;311: 62-71.

Brickwedde A, Brouwers N, van den Broek M et al. Structural, Physiological and Regulatory Analysis of Maltose Transporter Genes in Saccharomyces eubayanus CBS 12357T. Front Microbiol 2018;9. 
Brown CA, Murray AW, Verstrepen KJ. Rapid Expansion and Functional Divergence of Subtelomeric Gene Families in Yeasts. Curr Biol 2010;20: 895-903.

Bullis BL, Lemire BD. Isolation and characterization of the Saccharomyces cerevisiae SDH4 gene encoding a membrane anchor subunit of succinate dehydrogenase. J Biol Chem 1994;269: 6543-9.

Camarasa C, Faucet V, Dequin S. Role in anaerobiosis of the isoenzymes for Saccharomyces cerevisiae fumarate reductase encoded by OSM1 and FRDS1. Yeast 2007;24: 391-401.

Castegna A, Scarcia P, Agrimi G et al. Identification and functional characterization of a novel mitochondrial carrier for citrate and oxoglutarate in Saccharomyces cerevisiae. J Biol Chem 2010;285: 17359-70.

Castelli LM, Lui J, Campbell SG et al. Glucose depletion inhibits translation initiation via elF4A loss and subsequent $48 \mathrm{~S}$ preinitiation complex accumulation, while the pentose phosphate pathway is coordinately up-regulated. Mol Biol Cell 2011;22: 3379-93.

Cavero S, Traba J, Del Arco A et al. The calcium-dependent ATP-Mg/Pi mitochondrial carrier is a target of glucose-induced calcium signalling in Saccharomyces cerevisiae. Biochem J 2005;392: 537-44.

Chang YL, Hsieh MH, Chang WW et al. Instability of succinate dehydrogenase in SDHD polymorphism connects reactive oxygen species production to nuclear and mitochondrial genomic mutations in yeast. Antioxid Redox Signal 2015;22: 587-602.

Chapman KB, Solomon SD, Boeke JD. SDH1, the gene encoding the succinate dehydrogenase flavoprotein subunit from Saccharomyces cerevisiae. Gene 1992;118: 131-6.

Chaves RS, Herrero P, Ordiz I et al. Isocitrate lyase localisation in Saccharomyces cerevisiae cells. Gene 1997;198: 165-9.

Chen XJ. Sal1p, a calcium-cependent carrier protein that suppresses an essential cellular function associated with the Aac2 isoform of ADP/ATP translocase in Saccharomyces cerevisiae. Genetics 2004;167: 607-17.

Chen Y, Siewers V, Nielsen J. Profiling of cytosolic and peroxisomal acetyl-CoA metabolism in Saccharomyces cerevisiae. PloS one 2012;7: e42475.

Cho JH, Ha SJ, Kao LR et al. A novel DNA-binding protein bound to the mitochondrial inner membrane restores the null mutation of mitochondrial histone Abf2p in Saccharomyces cerevisiae. Mol Cell Biol 1998;18: 5712-23.

Colby G, Ishii Y, Tzagoloff A. Suppression of sdh1 mutations by the SDH1b gene of Saccharomyces cerevisiae. Yeast 1998;14: 1001-6.

Conant GC, Wolfe KH. Turning a hobby into a job: how duplicated genes find new functions. Nat Rev Genet 2008;9: 938-50.

Costanzo M, Baryshnikova A, Bellay J et al. The Genetic Landscape of a Cell. Science 2010;327: 425-31.

Costanzo M, VanderSluis B, Koch EN et al. A global genetic interaction network maps a wiring diagram of cellular function. Science 2016;353: aaf1420.

Cupp J, McAlister-Henn L. NAD (+)-dependent isocitrate dehydrogenase. Cloning, nucleotide sequence, and disruption of the IDH2 gene from Saccharomyces cerevisiae. J Biol Chem 1991;266: 22199205.

Cupp JR, McAlister-Henn L. Cloning and characterization of the gene encoding the IDH1 subunit of NAD (+)-dependent isocitrate dehydrogenase from Saccharomyces cerevisiae. J Biol Chem 1992;267: 16417-23.

de Jonge LP, Buijs NA, ten Pierick A et al. Scale-down of penicillin production in Penicillium chrysogenum. Biotechnol J 2011;6: 944-58.

DeLoache WC, Russ ZN, Dueber JE. Towards repurposing the yeast peroxisome for compartmentalizing heterologous metabolic pathways. Nat Commun 2016;7: 11152. 
Dickinson JR, Roy DJ, Dawes IW. A mutation affecting lipoamide dehydrogenase, pyruvate dehydrogenase and 2-oxoglutarate dehydrogenase activities in Saccharomyces cerevisiae. Mol Gen Genet 1986;204: 103-7.

Douma RD, de Jonge LP, Jonker CT et al. Intracellular metabolite determination in the presence of extracellular abundance: Application to the penicillin biosynthesis pathway in Penicillium chrysogenum. Biotechnol Bioeng 2010;107: 105-15.

Entian K-D, Kötter P. 25 Yeast Genetic Strain and Plasmid Collections. In: Stansfield I, Stark MJR (eds.) Methods in Microbiology volume 36: Academic Press, 2007, 629-66.

Eriksson P, André L, Ansell R et al. Cloning and characterization of GPD2, a second gene encoding snglycerol 3-phosphate dehydrogenase (NAD+) in Saccharomyces cerevisiae, and its comparison with GPD1. Mol Microbiol 1995;17: 95-107.

Escalera-Fanjul X, Quezada H, Riego-Ruiz L et al. Whole-Genome Duplication and Yeast's Fruitful Way of Life. Trends Genet 2019;35: 42-54.

Fares MA, Keane OM, Toft C et al. The roles of whole-genome and small-scale duplications in the functional specialization of Saccharomyces cerevisiae genes. PLoS Genet 2013;9: e1003176.

Fazius F, Shelest E, Gebhardt $P$ et al. The fungal $\alpha$-aminoadipate pathway for lysine biosynthesis requires two enzymes of the aconitase family for the isomerization of homocitrate to homoisocitrate. Mol Microbiol 2012;86: 1508-30.

Fernández $\mathrm{E}$, Fernández $\mathrm{M}$, Rodicio R. Two structural genes are encoding malate synthase isoenzymes in Saccharomyces cerevisiae. FEBS Lett 1993;320: 271-5.

Fernández E, Moreno F, Rodicio R. The ICL1 gene from Saccharomyces cerevisiae. Eur J Biochem 1992;204: 983-90.

Fernández M, Fernández E, Rodicio R. ACR1, a gene encoding a protein related to mitochondrial carriers, is essential for acetyl-CoA synthetase activity in Saccharomyces cerevisiae. Mol Gen Genet 1994;242: 727-35.

François JM, Lachaux C, Morin N. Synthetic Biology Applied to Carbon Conservative and Carbon Dioxide Recycling Pathways. Front Bioeng Biotechnol 2020;7.

Gancedo C, Flores CL. Moonlighting proteins in yeasts. Microbiol Mol Biol Rev 2008;72: 197-210.

Gangloff SP, Marguet D, Lauquin G. Molecular cloning of the yeast mitochondrial aconitase gene (ACO1) and evidence of a synergistic regulation of expression by glucose plus glutamate. $\mathrm{Mol} \mathrm{Cell} \mathrm{Biol}$ 1990;10: 3551-61.

Gawaz M, Douglas MG, Klingenberg M. Structure-function studies of adenine nucleotide transport in mitochondria. II. Biochemical analysis of distinct AAC1 and AAC2 proteins in yeast. J Biol Chem 1990;265: 14202-8.

Gebert N, Gebert M, Oeljeklaus S et al. Dual function of Sdh3 in the respiratory chain and TIM22 protein translocase of the mitochondrial inner membrane. Mol Cell 2011;44: 811-8.

Giaever G, Chu AM, Ni L et al. Functional profiling of the Saccharomyces cerevisiae genome. Nature 2002;418: 387-91.

Gietz RD, Woods RA. Transformation of yeast by lithium acetate/single-stranded carrier DNA/polyethylene glycol method. Methods Enzymol 2002;350: 87-96.

Hartig A, Simon MM, Schuster T et al. Differentially regulated malate synthase genes participate in carbon and nitrogen metabolism of S. cerevisiae. Nucleic Acids Res 1992;20: 5677-86.

Haselbeck RJ, Colman RF, McAlister-Henn L. Isolation and sequence of a cDNA encoding porcine mitochondrial NADP-specific isocitrate dehydrogenase. Biochemistry 1992;31: 6219-23.

Hashimoto M, Ichimura T, Mizoguchi $\mathrm{H}$ et al. Cell size and nucleoid organization of engineered Escherichia coli cells with a reduced genome. Mol Microbiol 2005;55: 137-49.

Herzig S, Raemy E, Montessuit S et al. Identification and functional expression of the mitochondrial pyruvate carrier. Science 2012;337: 93-6. 
Hiltunen JK, Mursula AM, Rottensteiner $\mathrm{H}$ et al. The biochemistry of peroxisomal beta-oxidation in the yeast Saccharomyces cerevisiae. FEMS Microbiol Rev 2003;27: 35-64.

Hutchison CA, Chuang R-Y, Noskov VN et al. Design and synthesis of a minimal bacterial genome. Science 2016;351: aad6253.

Jansen MLA, Bracher JM, Papapetridis I et al. Saccharomyces cerevisiae strains for second-generation ethanol production: from academic exploration to industrial implementation. FEMS Yeast Res 2017;17: fox044.

Jia YK, Bécam AM, Herbert C. The CIT3 gene of Saccharomyces cerevisiae encodes a second mitochondrial isoform of citrate synthase. Mol Microbiol 1997;24: 53-9.

Jouhten P, Penttilä M. Anaerobic Carbon Metabolism of Saccharomyces cerevisiae. In: Piškur J, Compagno C (eds.) Molecular Mechanisms in Yeast Carbon Metabolism, DOI 10.1007/978-3642-55013-3_3. Berlin, Heidelberg: Springer Berlin Heidelberg, 2014, 57-82.

Kaplan RS, Mayor JA, Gremse DA et al. High level expression and characterization of the mitochondrial citrate transport protein from the yeast Saccharomyces cerevisiae. J Biol Chem 1995;270: 410814.

Kaplan RS, Mayor JA, Kakhniashvili D et al. Deletion of the nuclear gene encoding the mitochondrial citrate transport protein from Saccharomyces cerevisiae. Biochem Biophys Res Commun 1996;226: 657-62.

Kim KS, Rosenkrantz MS, Guarente L. Saccharomyces cerevisiae contains two functional citrate synthase genes. Mol Cell Biol 1986;6: 1936-42.

Knijnenburg TA, Daran J-MG, van den Broek MA et al. Combinatorial effects of environmental parameters on transcriptional regulation in Saccharomyces cerevisiae: a quantitative analysis of a compendium of chemostat-based transcriptome data. BMC genomics 2009;10: 53.

Kolarov J, Kolarova N, Nelson N. A third ADP/ATP translocator gene in yeast. J Biol Chem 1990;265: 12711-6.

Komatsu M, Uchiyama T, Omura S et al. Genome-minimized Streptomyces host for the heterologous expression of secondary metabolism. Proc Natl Acad Sci U S A 2010;107: 2646-51.

Koopman F, Beekwilder J, Crimi B et al. De novo production of the flavonoid naringenin in engineered Saccharomyces cerevisiae. Microb Cell Fact 2012;11: 155.

Koren S, Walenz BP, Berlin K et al. Canu: scalable and accurate long-read assembly via adaptive k-mer weighting and repeat separation. Genome Res 2017;27: 722-36.

Krivoruchko A, Zhang Y, Siewers $\mathrm{V}$ et al. Microbial acetyl-CoA metabolism and metabolic engineering. Metab Eng 2015;28: 28-42.

Kuijpers NG, Solis-Escalante D, Luttik MA et al. Pathway swapping: Toward modular engineering of essential cellular processes. Proc Natl Acad Sci USA 2016;113: 15060-5.

Kunze M, Kragler F, Binder M et al. Targeting of malate synthase 1 to the peroxisomes of Saccharomyces cerevisiae cells depends on growth on oleic acid medium. Eur J Biochem 2002;269: 915-22.

Kunze M, Pracharoenwattana I, Smith SM et al. A central role for the peroxisomal membrane in glyoxylate cycle function. Biochim Biophys Acta 2006;1763: 1441-52.

Kurita O, Nishida Y. Involvement of mitochondrial aldehyde dehydrogenase ALD5 in maintenance of the mitochondrial electron transport chain in Saccharomyces cerevisiae. FEMS Microbiol Lett 1999;181: 281-7.

Kuzmin E, VanderSluis B, Nguyen Ba AN et al. Exploring whole-genome duplicate gene retention with complex genetic interaction analysis. Science 2020;368: eaaz5667.

Kuzmin E, VanderSluis B, Wang W et al. Systematic analysis of complex genetic interactions. Science 2018;360: eaao1729.

Laco J, Zeman I, Pevala V et al. Adenine nucleotide transport via Sal1 carrier compensates for the essential function of the mitochondrial ADP/ATP carrier. FEMS Yeast Res 2010;10: 290-6. 
Lara AR, Gosset G. Minimal Cells: Design, Construction, Biotechnological Applications, DOI https://doi.org/10.1007/978-3-030-31897-0: Springer International Publishing, 2019, 230.

Lee YJ, Jang JW, Kim KJ et al. TCA cycle-independent acetate metabolism via the glyoxylate cycle in Saccharomyces cerevisiae. Yeast 2011;28: 153-66.

Li H, Durbin R. Fast and accurate short read alignment with Burrows-Wheeler transform. Bioinformatics 2009;25: 1754-60.

Li H, Handsaker B, Wysoker A et al. The Sequence Alignment/Map format and SAMtools. Bioinformatics 2009;25: 2078-9.

Lieder S, Nikel PI, de Lorenzo V et al. Genome reduction boosts heterologous gene expression in Pseudomonas putida. Microb Cell Fact 2015;14: 23.

Liu Q, Yu T, Li X et al. Rewiring carbon metabolism in yeast for high level production of aromatic chemicals. Nat Commun 2019;10: 4976.

Liu Z, Österlund T, Hou J et al. Anaerobic $\alpha$-amylase production and secretion with fumarate as the final electron acceptor in Saccharomyces cerevisiae. Appl Environ Microbiol 2013;79: 2962-7.

Lobo Z, Maitra PK. Pentose phosphate pathway mutants of yeast. Mol Gen Genet 1982;185: 367-8.

Lombardo A, Carine K, Scheffler IE. Cloning and characterization of the iron-sulfur subunit gene of succinate dehydrogenase from Saccharomyces cerevisiae. J Biol Chem 1990;265: 10419-23.

Looke M, Kristjuhan K, Kristjuhan A. Extraction of genomic DNA from yeasts for PCR-based applications. BioTechniques 2011;50: 325-8.

Luo Z, Yu K, Xie S et al. Compacting a synthetic yeast chromosome arm. Genome Biol 2021;22: 5.

Luttik MA, Kötter P, Salomons FA et al. The Saccharomyces cerevisiae ICL2 gene encodes a mitochondrial 2-methylisocitrate lyase involved in propionyl-coenzyme A metabolism. J Bacteriol 2000;182: 7007-13.

Mans R, van Rossum HM, Wijsman M et al. CRISPR/Cas9: a molecular Swiss army knife for simultaneous introduction of multiple genetic modifications in Saccharomyces cerevisiae. FEMS Yeast Res 2015;15: 1-15.

Marobbio CM, Vozza A, Harding $M$ et al. Identification and reconstitution of the yeast mitochondrial transporter for thiamine pyrophosphate. Embo j 2002;21: 5653-61.

Marobbio CMT, Giannuzzi G, Paradies E et al. alpha-Isopropylmalate, a leucine biosynthesis intermediate in yeast, is transported by the mitochondrial oxalacetate carrier. J Biol Chem 2008;283: 28445-53.

Matsushika A, Goshima T, Fujii T et al. Characterization of non-oxidative transaldolase and transketolase enzymes in the pentose phosphate pathway with regard to xylose utilization by recombinant Saccharomyces cerevisiae. Enzyme Microb Technol 2012;51: 16-25.

Meaden PG, Dickinson FM, Mifsud A et al. The ALD6 gene of Saccharomyces cerevisiae encodes a cytosolic, $\mathrm{Mg}(2+)$-activated acetaldehyde dehydrogenase. Yeast 1997;13: 1319-27.

Meadows AL, Hawkins KM, Tsegaye $Y$ et al. Rewriting yeast central carbon metabolism for industrial isoprenoid production. Nature 2016;537: 694-7.

Minard KI, McAlister-Henn L. Isolation, nucleotide sequence analysis, and disruption of the MDH2 gene from Saccharomyces cerevisiae: evidence for three isozymes of yeast malate dehydrogenase. Mol Cell Biol 1991;11: 370-80.

Miyagi H, Kawai S, Murata K. Two sources of mitochondrial NADPH in the yeast Saccharomyces cerevisiae. J Biol Chem 2009;284: 7553-60.

Morimoto $\mathrm{T}$, Kadoya R, Endo $\mathrm{K}$ et al. Enhanced recombinant protein productivity by genome reduction in Bacillus subtilis. DNA Res 2008;15: 73-81.

Murakami K, Tao E, Ito Y et al. Large scale deletions in the Saccharomyces cerevisiae genome create strains with altered regulation of carbon metabolism. Appl Microbiol Biotechnol 2007;75: 58997. 
Mutschler H, Robinson T, Tang TD et al. Special Issue on Bottom-Up Synthetic Biology. Chembiochem 2019;20: 2533-4.

Navarro-Aviño JP, Prasad R, Miralles VJ et al. A proposal for nomenclature of aldehyde dehydrogenases in Saccharomyces cerevisiae and characterization of the stress-inducible ALD2 and ALD3 genes. Yeast 1999;15: 829-42.

Neal SE, Dabir DV, Wijaya J et al. Osm1 facilitates the transfer of electrons from Erv1 to fumarate in the redox-regulated import pathway in the mitochondrial intermembrane space. Mol Biol Cell 2017;28: 2773-85.

Nevoigt E, Stahl U. Osmoregulation and glycerol metabolism in the yeast Saccharomyces cerevisiae. FEMS Microbiol Rev 1997;21: 231-41.

Niedenführ S, ten Pierick A, van Dam PT et al. Natural isotope correction of MS/MS measurements for metabolomics and (13)C fluxomics. Biotechnol Bioeng 2016;113: 1137-47.

Nielsen J, Keasling Jay D. Engineering Cellular Metabolism. Cell 2016;164: 1185-97.

Nijkamp JF, van den Broek M, Datema E et al. De novo sequencing, assembly and analysis of the genome of the laboratory strain Saccharomyces cerevisiae CEN.PK113-7D, a model for modern industrial biotechnology. Microb Cell Fact 2012a;11: 36.

Nijkamp JF, van den Broek MA, Geertman JM et al. De novo detection of copy number variation by coassembly. Bioinformatics 2012b;28: 3195-202.

Nissen TL, Hamann CW, Kielland-Brandt MC et al. Anaerobic and aerobic batch cultivations of Saccharomyces cerevisiae mutants impaired in glycerol synthesis. Yeast 2000;16: 463-74.

Norbeck J, Pâhlman AK, Akhtar N et al. Purification and characterization of two isoenzymes of DLglycerol-3-phosphatase from Saccharomyces cerevisiae. Identification of the corresponding GPP1 and GPP2 genes and evidence for osmotic regulation of Gpp2p expression by the osmosensing mitogen-activated protein kinase signal transduction pathway. J Biol Chem 1996;271: 13875-81.

Orlandi I, Casatta N, Vai M. Lack of Ach1 CoA-Transferase Triggers Apoptosis and Decreases Chronological Lifespan in Yeast. Front Oncol 2012;2: 67.

Pahlman AK, Granath K, Ansell R et al. The yeast glycerol 3-phosphatases Gpp1p and Gpp2p are required for glycerol biosynthesis and differentially involved in the cellular responses to osmotic, anaerobic, and oxidative stress. J Biol Chem 2001;276: 3555-63.

Palmieri F, Agrimi G, Blanco E et al. Identification of mitochondrial carriers in Saccharomyces cerevisiae by transport assay of reconstituted recombinant proteins. Biochim et Biophys Acta 2006;1757: 1249-62.

Palmieri F, Monné M. Discoveries, metabolic roles and diseases of mitochondrial carriers: A review. Biochim Biophys Acta 2016;1863: 2362-78.

Palmieri L, Agrimi G, Runswick MJ et al. Identification in Saccharomyces cerevisiae of two isoforms of a novel mitochondrial transporter for 2-oxoadipate and 2-oxoglutarate. J Biol Chem 2001;276: 1916-22.

Palmieri L, Lasorsa FM, De Palma A et al. Identification of the yeast ACR1 gene product as a succinatefumarate transporter essential for growth on ethanol or acetate. FEBS Lett 1997;417: 114-8.

Palmieri L, Palmieri F, Runswick MJ et al. Identification by bacterial expression and functional reconstitution of the yeast genomic sequence encoding the mitochondrial dicarboxylate carrier protein. FEBS Lett 1996;399: 299-302.

Palmieri L, Vozza A, Agrimi G et al. Identification of the yeast mitochondrial transporter for oxaloacetate and sulfate. J Biol Chem 1999a;274: 22184-90.

Palmieri L, Vozza A, Hönlinger A et al. The mitochondrial dicarboxylate carrier is essential for the growth of Saccharomyces cerevisiae on ethanol or acetate as the sole carbon source. Mol Microbiol 1999b;31: 569-77. 
Papagianni M. Recent advances in engineering the central carbon metabolism of industrially important bacteria. Microb Cell Fact 2012;11: 50.

Postma ED, Dashko S, van Breemen L et al. A supernumerary designer chromosome for modular in vivo pathway assembly in Saccharomyces cerevisiae. Nucleic Acids Res 2021;49: 1769-83.

Prohl C, Pelzer W, Diekert K et al. The yeast mitochondrial carrier Leu5p and its human homologue Graves' disease protein are required for accumulation of coenzyme A in the matrix. Mol Cell Biol 2001;21: 1089-97.

Pronk JT, Yde Steensma H, Van Dijken JP. Pyruvate metabolism in Saccharomyces cerevisiae. Yeast 1996;12: 1607-33.

Przybyla-Zawislak B, Dennis RA, Zakharkin SO et al. Genes of succinyl-CoA ligase from Saccharomyces cerevisiae. Eur J Biochem 1998;258: 736-43.

Regev-Rudzki N, Karniely S, Ben-Haim NN et al. Yeast aconitase in two locations and two metabolic pathways: seeing small amounts is believing. Mol Biol Cell 2005;16: 4163-71.

Repetto $B$, Tzagoloff A. Structure and regulation of $K G D 1$, the structural gene for yeast alphaketoglutarate dehydrogenase. Mol Cell Biol 1989;9: 2695-705.

Repetto B, Tzagoloff A. Structure and regulation of $K G D 2$, the structural gene for yeast dihydrolipoyl transsuccinylase. Mol Cell Biol 1990;10: 4221-32.

Richardson SM, Mitchell LA, Stracquadanio G et al. Design of a synthetic yeast genome. Science 2017;355: 1040-4.

Salazar AN, Gorter de Vries AR, van den Broek M et al. Nanopore sequencing enables near-complete de novo assembly of Saccharomyces cerevisiae reference strain CEN.PK113-7D. FEMS Yeast Res 2017;17.

Sánchez-Pascuala A, Fernández-Cabezón L, de Lorenzo V et al. Functional implementation of a linear glycolysis for sugar catabolism in Pseudomonas putida. Metab Eng 2019;54: 200-11.

Sasaki M, Kumagai H, Takegawa K et al. Characterization of genome-reduced fission yeast strains. Nucleic Acids Res 2013;41: 5382-99.

Scarcia P, Palmieri L, Agrimi G et al. Three mitochondrial transporters of Saccharomyces cerevisiae are essential for ammonium fixation and lysine biosynthesis in synthetic minimal medium. $\mathrm{Mol}$ Genet Metab 2017;122: 54-60.

Schaaff-Gerstenschläger I, Mannhaupt G, Vetter I et al. TKL2, a second transketolase gene of Saccharomyces cerevisiae. Eur J Biochem 1993;217: 487-92.

Shao Y, Lu N, Wu Z et al. Creating a functional single-chromosome yeast. Nature 2018;560: 331-5.

Shen X-X, Opulente DA, Kominek J et al. Tempo and Mode of Genome Evolution in the Budding Yeast Subphylum. Cell 2018;175: 1533-45.e20.

Smith CP, Thorsness PE. The molecular basis for relative physiological functionality of the ADP/ATP carrier isoforms in Saccharomyces cerevisiae. Genetics 2008;179: 1285-99.

Solis-Escalante D, Kuijpers NG, Barrajon-Simancas $\mathrm{N}$ et al. A minimal set of glycolytic genes reveals strong redundancies in Saccharomyces cerevisiae central metabolism. Eukaryot Cell 2015;14: 804-16.

Solis-Escalante D, Kuijpers NG, Bongaerts $\mathrm{N}$ et al. amdSYM, a new dominant recyclable marker cassette for Saccharomyces cerevisiae. FEMS Yeast Res 2013;13: 126-39.

Soontorngun N, Larochelle M, Drouin S et al. Regulation of gluconeogenesis in Saccharomyces cerevisiae is mediated by activator and repressor functions of Rds2. Mol Cell Biol 2007;27: 7895-905.

Stanford D, Whitney M, Hurto R et al. Division of labor among the yeast Sol proteins implicated in tRNA nuclear export and carbohydrate metabolism. Genetics 2004;168: 117-27.

Stucka R, Dequin S, Salmon J-M et al. DNA sequences in chromosomes 11 and VII code for pyruvate carboxylase isoenzymes in Saccharomyces cerevisiae: analysis of pyruvate carboxylase-deficient strains. Mol Gen Genet 1991;229: 307-15. 
Suzuki N, Nonaka H, Tsuge Y et al. New multiple-deletion method for the Corynebacterium glutamicum genome, using a mutant lox sequence. Appl Environ Microbiol 2005;71: 8472-80.

Swiegers JH, Dippenaar N, Pretorius IS et al. Carnitine-dependent metabolic activities in Saccharomyces cerevisiae: three carnitine acetyltransferases are essential in a carnitine-dependent strain. Yeast 2001;18: 585-95.

Szeto SS, Reinke SN, Oyedotun KS et al. Expression of Saccharomyces cerevisiae Sdh3p and Sdh4p paralogs results in catalytically active succinate dehydrogenase isoenzymes. $J$ Biol Chem 2012;287: 22509-20.

Takahashi H, McCaffery JM, Irizarry RA et al. Nucleocytosolic acetyl-coenzyme a synthetase is required for histone acetylation and global transcription. Mol Cell 2006;23: 207-17.

Taylor KM, Kaplan CP, Gao X et al. Localization and targeting of isocitrate lyases in Saccharomyces cerevisiae. Biochem J 1996;319: 255-62.

Thorvaldsdottir H, Robinson JT, Mesirov JP. Integrative Genomics Viewer (IGV): high-performance genomics data visualization and exploration. Brief Bioinform 2013;14: 178-92.

Tibbetts AS, Sun Y, Lyon NA et al. Yeast mitochondrial oxodicarboxylate transporters are important for growth on oleic acid. Arch Biochem Biophys 2002;406: 96-104.

Timón-Gómez A, Proft M, Pascual-Ahuir A. Differential regulation of mitochondrial pyruvate carrier genes modulates respiratory capacity and stress tolerance in yeast. PloS one 2013;8: e79405.

Todisco S, Agrimi G, Castegna A et al. Identification of the mitochondrial NAD+ transporter in Saccharomyces cerevisiae. J Biol Chem 2006;281: 1524-31.

Tong AHY, Evangelista M, Parsons AB et al. Systematic Genetic Analysis with Ordered Arrays of Yeast Deletion Mutants. Science 2001;294: 2364-8.

Tong AHY, Lesage G, Bader GD et al. Global Mapping of the Yeast Genetic Interaction Network. Science 2004;303: 808-13.

Tzagoloff A, Jang J, Glerum DM et al. FLX1 codes for a carrier protein involved in maintaining a proper balance of flavin nucleotides in yeast mitochondria. J Biol Chem 1996;271: 7392-7.

Valdés-Hevia MD, de la Guerra R, Gancedo C. Isolation and characterization of the gene encoding phosphoenolpyruvate carboxykinase from Saccharomyces cerevisiae. FEBS Lett 1989;258: 313-6.

Van Dijken JP, Scheffers WA. Redox balances in the metabolism of sugars by yeasts. FEMS Microbiol Rev 1986;1: 199-224.

van Rossum HM, Kozak BU, Niemeijer MS et al. Alternative reactions at the interface of glycolysis and citric acid cycle in Saccharomyces cerevisiae. FEMS Yeast Res 2016a;16.

van Rossum HM, Kozak BU, Pronk JT et al. Engineering cytosolic acetyl-coenzyme A supply in Saccharomyces cerevisiae: Pathway stoichiometry, free-energy conservation and redox-cofactor balancing. Metab Eng 2016b;36: 99-115.

Verduyn C, Postma E, Scheffers WA et al. Effect of benzoic acid on metabolic fluxes in yeasts: A continuous-culture study on the regulation of respiration and alcoholic fermentation. Yeast 1992;8: 501-17.

Walker BJ, Abeel T, Shea T et al. Pilon: an integrated tool for comprehensive microbial variant detection and genome assembly improvement. PloS one 2014;9: e112963.

White WH, Skatrud PL, Xue Z et al. Specialization of function among aldehyde dehydrogenases: the ALD2 and ALD3 genes are required for beta-alanine biosynthesis in Saccharomyces cerevisiae. Genetics 2003;163: 69-77.

Wieczorke R, Krampe S, Weierstall T et al. Concurrent knock-out of at least 20 transporter genes is required to block uptake of hexoses in Saccharomyces cerevisiae. FEBS Lett 1999;464: 123-8.

Wijsman M, Swiat MA, Marques WL et al. A toolkit for rapid CRISPR-SpCas9 assisted construction of hexose-transport-deficient Saccharomyces cerevisiae strains. FEMS Yeast Res 2019;19: foy107. 
Winzeler EA, Shoemaker DD, Astromoff A et al. Functional characterization of the S. cerevisiae genome by gene deletion and parallel analysis. Science 1999;285: 901-6.

Wisselink HW, Cipollina C, Oud B et al. Metabolome, transcriptome and metabolic flux analysis of arabinose fermentation by engineered Saccharomyces cerevisiae. Metab Eng 2010;12: 537-51.

Wu L, Mashego MR, van Dam JC et al. Quantitative analysis of the microbial metabolome by isotope dilution mass spectrometry using uniformly $13 \mathrm{C}$-labeled cell extracts as internal standards. Anal Biochem 2005;336: 164-71.

Xiberras J, Klein M, Nevoigt E. Glycerol as a substrate for Saccharomyces cerevisiae based bioprocesses Knowledge gaps regarding the central carbon catabolism of this 'non-fermentable' carbon source. Biotechnol Adv 2019;37: 107378.

Zhao WN, McAlister-Henn L. Expression and gene disruption analysis of the isocitrate dehydrogenase family in yeast. Biochemistry 1996;35: 7873-8.

Zhu D, Fu Y, Liu F et al. Enhanced heterologous protein productivity by genome reduction in Lactococcus lactis NZ9000. Microb Cell Fact 2017;16: 1. 


\section{Acknowledgements}

We thank Pilar de la Torre and Marcel van den Broek for sequencing and bioinformatic analysis, Roel Sarelse, Ilse Pardijs and Lycka Kamoen for strain construction, Koen Verhagen, Patricia van Dam and Martin Pabst for analysis of intracellular metabolites, Erik de Hulster for assistance with sampling of the bioreactors, Marijke Luttik for the growth experiment on pyruvate medium, Sofia Dashko and Jean-Marc Daran for input during the literature analysis and Jack Pronk for feedback on an advanced version of the manuscript. This work was funded by the AdLibYeast ERC consolidator grant number 648141 attributed to P.D.-L. 


\section{Figure 1 - CCM precursors of industrially relevant chemicals}

Abbreviations: 3-PG is 3-Phosphoglycerate and PEP is phosphoenolpyruvate.

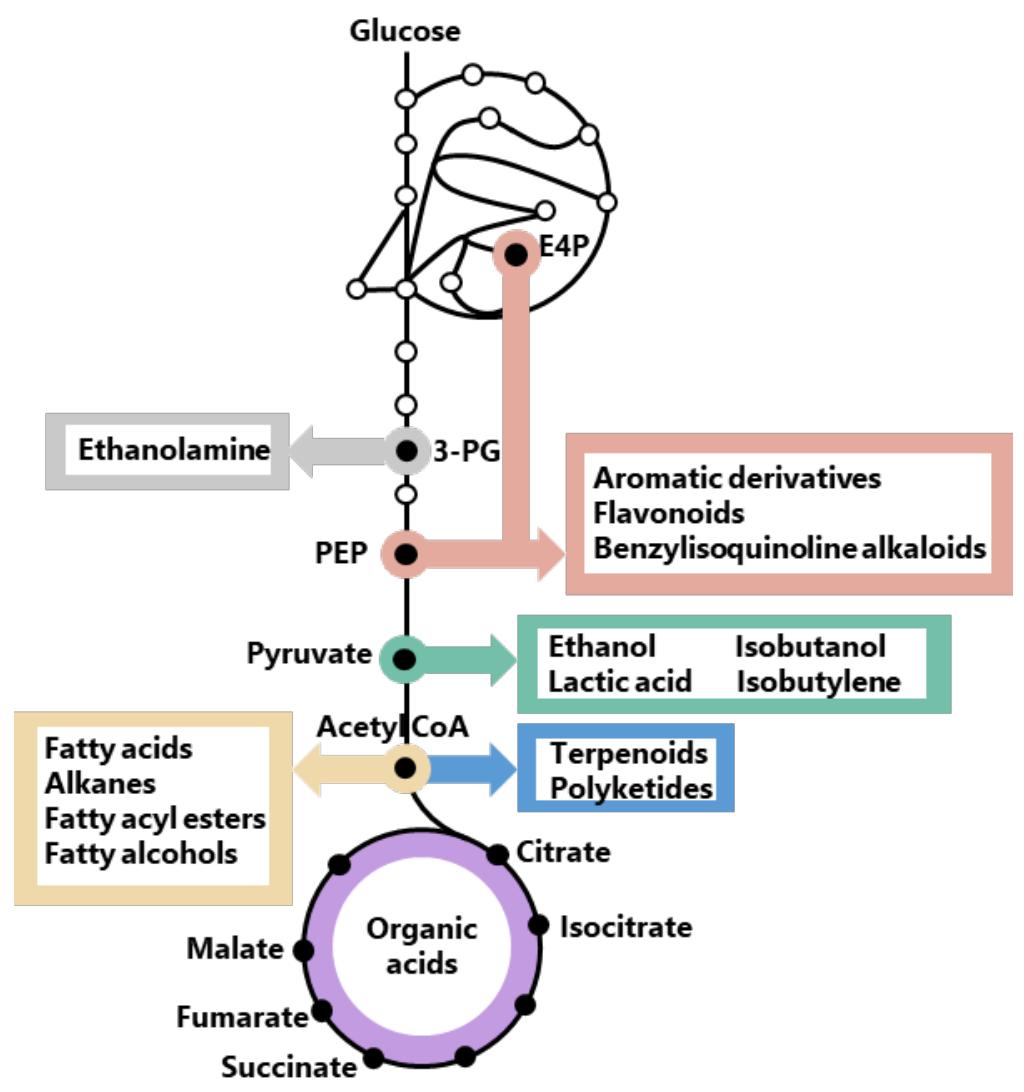




\section{Figure 2 - Reactions of the central carbon metabolism (CCM) of $S$.} cerevisiae considered for genetic reduction

Schematic representation of sub-processes and pathways in CCM metabolic pathways considered for genetic reduction in this study. Enzyme-catalysed conversions (black lines) and transport processes (dotted lines) are shown between intermediates and through mitochondrial transporters (circles and ovals), respectively. Directionality and reversibility of reactions was based on https://pathway.yeastgenome.org/. Enzyme localization was based on literature information. Genes retained in the genetic reduction strategy are shown in black, genes selected for deletion in the minimal CCM strain are indicated in bold and underlined. Occurrence of pathways in different cellular compartments is shown by grey borders. Simplifications have been made for visualization reasons, for example $\mathrm{H}_{2} \mathrm{O}$ and inorganic phosphate are not shown. Abbreviations can be found in the abbreviation list. 
bioRxiv preprint doi: https://doi.org/10.1101/2021.08.24.457526; this version posted August 26, 2021. The copyright holder for this preprint (which was not certified by peer review) is the author/funder, who has granted bioRxiv a license to display the preprint in perpetuity. It is made available under aCC-BY-NC-ND 4.0 International license.

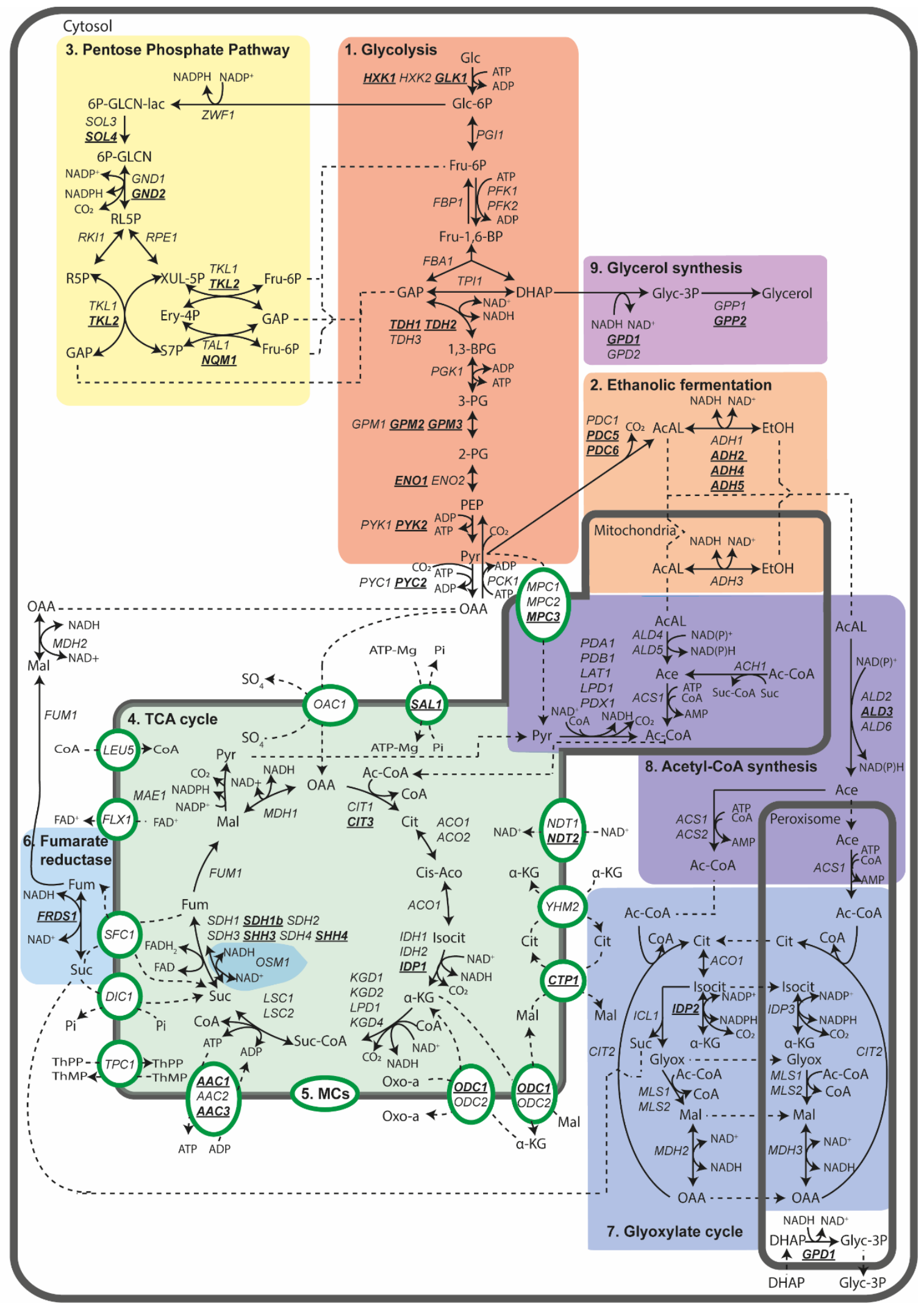




\section{Figure 3 - Deletion strategy and specific growth rates of resulting strains}

Construction workflow for relevant $S$. cerevisiae strains $(A)$ and their respective specific growth rates measured in shake flask rate on synthetic medium with glucose (SMD) or ethanol (SME) as carbon source, supplemented with uracil (B). Specific growth rates represent average and standard deviation of measurements on independent duplicate cultures for each strain and are expressed as a percentage of specific growth rate of the naïve uracil auxotrophic reference strain S. cerevisiae IMX581 or the naïve uracil prototrophic reference strain S. cerevisiae CEN.PK113-7D. Significant differences in specific growth rate relative to the control strain are indicated with a * (two-tailed paired homoscedastic t-test $p<0.05$ ). 
A)

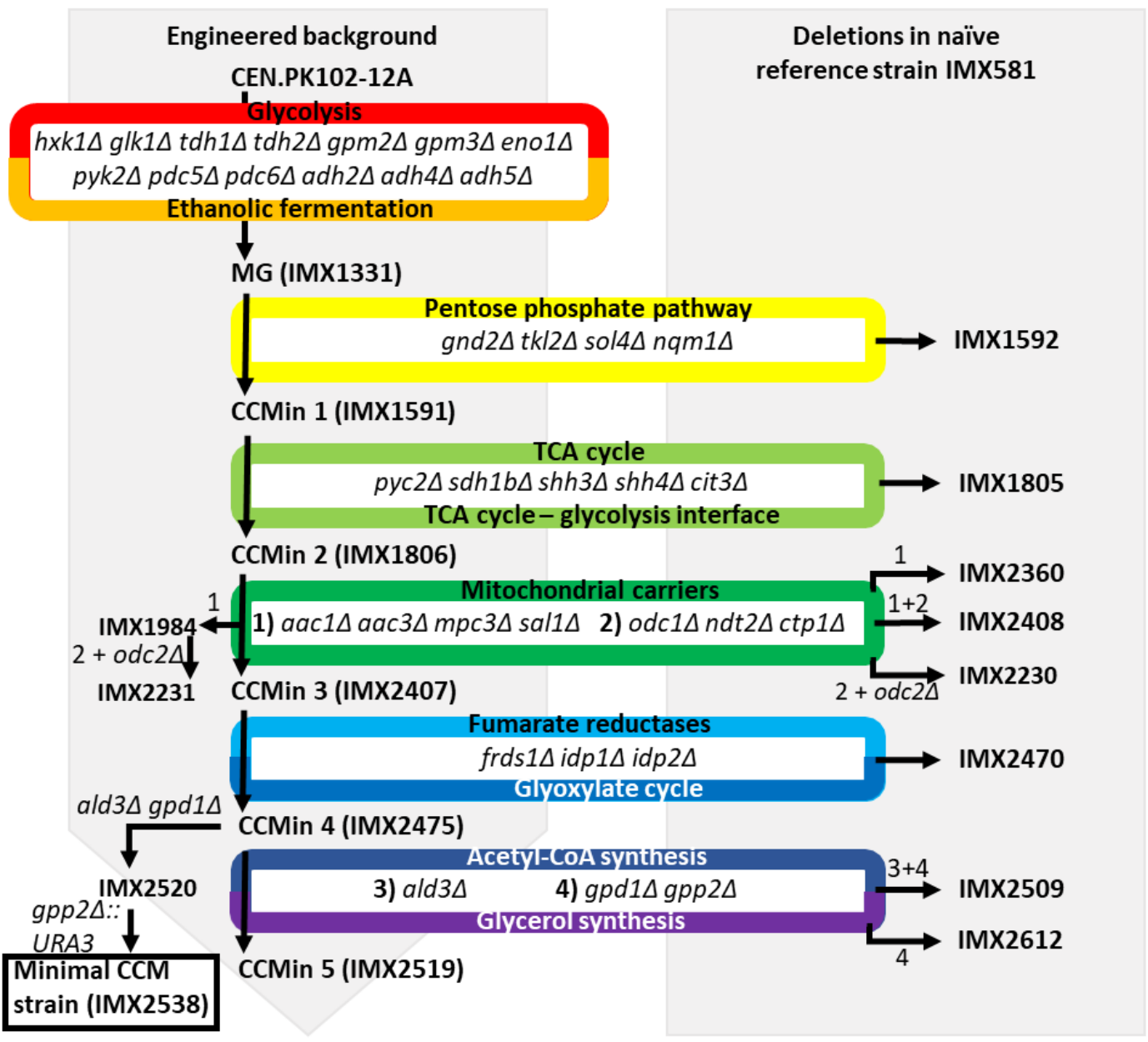

B) Engineered background, Glucose

Naïve background, Glucose
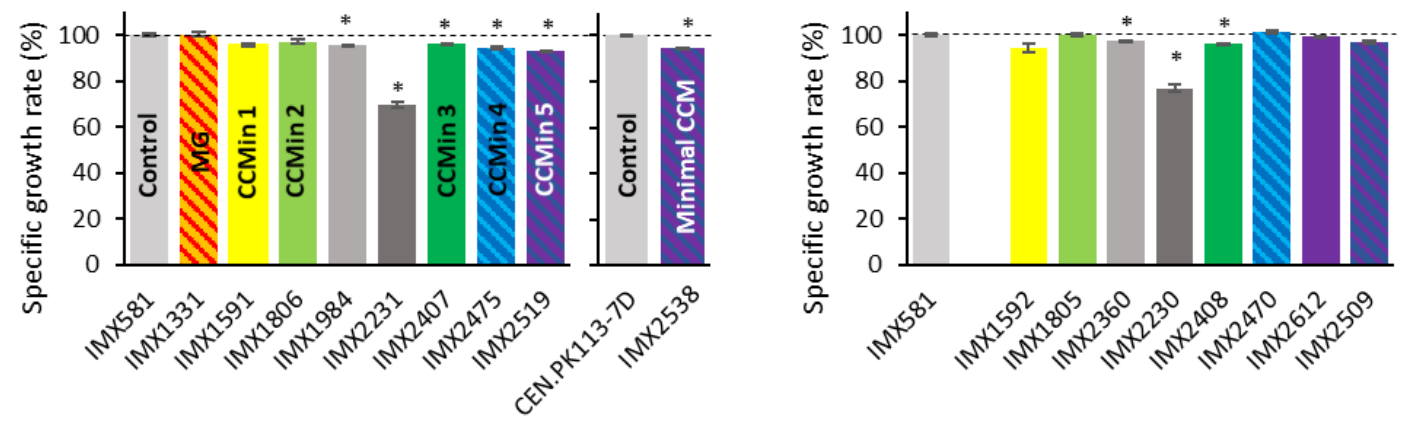

Engineered background, Ethanol

Naïve background, Ethanol
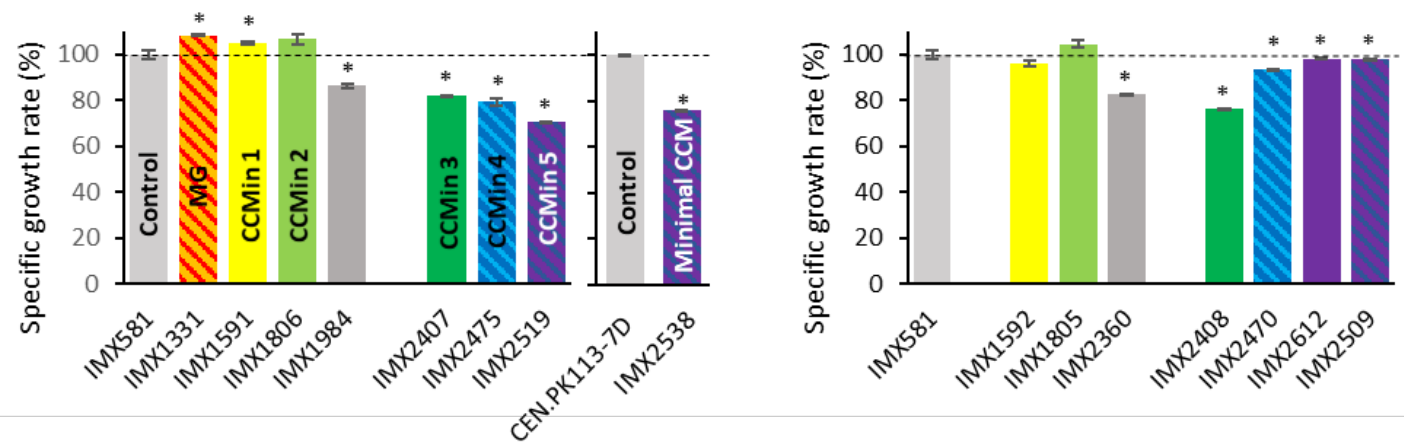


\section{Figure 4-Specific growth rates of 35-deletion, prototrophic minimal CCM}

\section{strain under a broad range of growth conditions}

Specific growth rates of the prototrophic S. cerevisiae strains CEN.PK113-7D (naïve reference strain), IMX372 (minimal glycolysis (MG)) and IMX2538 (minimal CCM) under different growth conditions. Specific growth rates were measured in triplicate cultures using a growth profiler, except for those in SMPyr and SMD-anaerobic, which were measured in independent duplicate shake flask cultures. Abbreviations indicate the following growth conditions: SM, synthetic medium; SMD, synthetic medium with glucose; Gal, galactose as carbon source; Mal, maltose as carbon source; Suc, sucrose as carbon source; Fruc, fructose as carbon source; Pyr, pyruvate as carbon source; YPD, complex medium with glucose. Significant changes in growth rate of IMX372 and IMX2538 with respect to CEN.PK113-7D are indicated with a * (twotailed paired homoscedastic $t$-test $p<0.05)$.

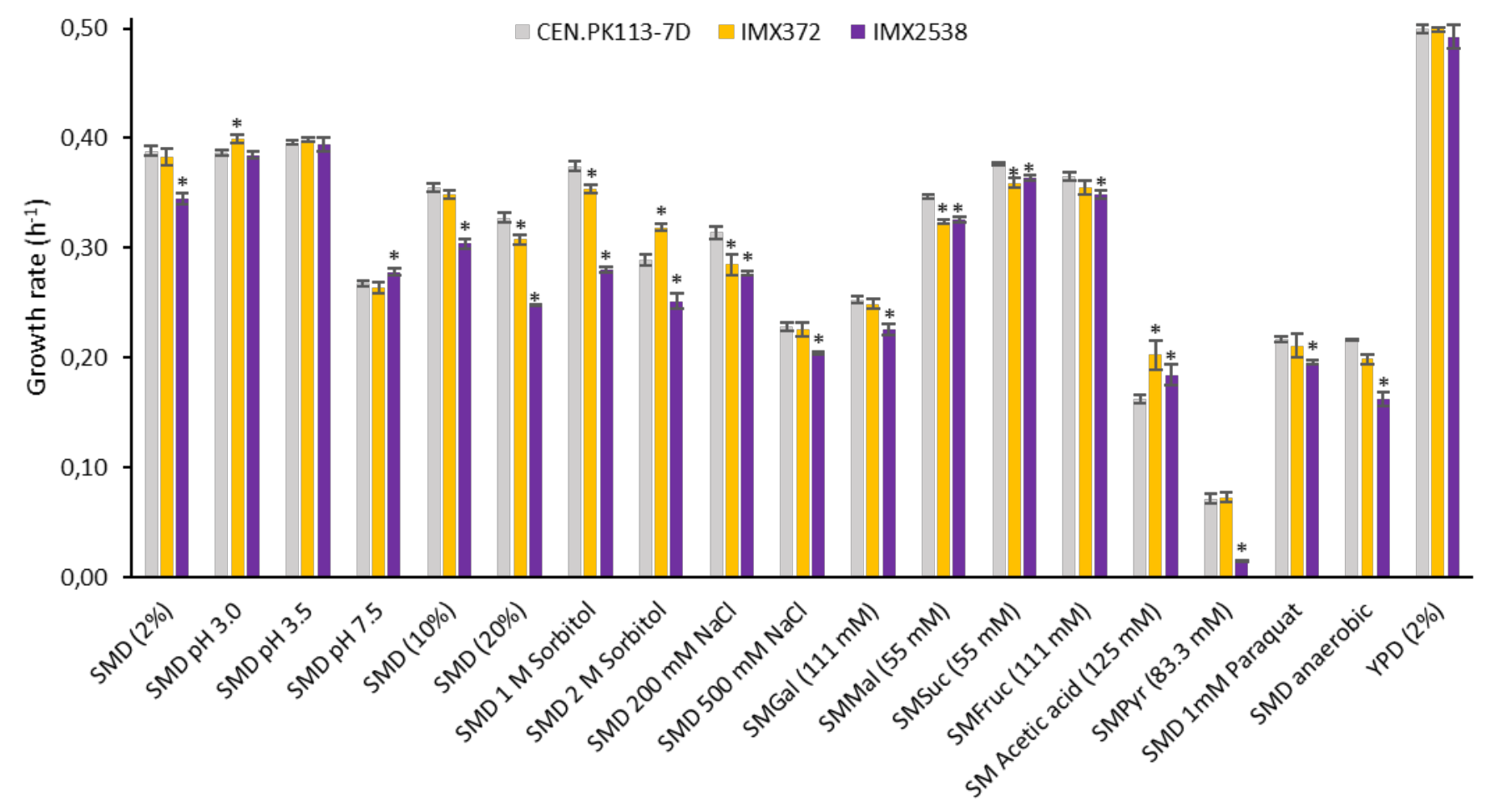




\section{Table 1 Single-nucleotide mutations identified in coding regions of the} prototrophic minimal CCM strain IMX2538

Single-nucleotide changes in S. cerevisiae IMX2538 (prototrophic minimal CCM) relative to the genome sequence of S. cerevisiae IMX372 (prototrophic minimal glycolysis (MG)) (Solis-Escalante et al. 2015).

\begin{tabular}{llll}
\hline Systematic name & Name & Type* & Amino acid change \\
\hline YBR114W & RAD16 & NS & Ile-202-Thr \\
YDL035C & GPR1 & S & Asn-523-Asn \\
YDR098C & GRX3 & NS & Glu-239-Asp \\
YFL062W & COS4 & S & Cys-151-Cys \\
YLR002C & NOC3 & NS & Asp-526-Glu \\
YML058W & SML1 & NS & Gly-52-Ser \\
YMR154C & RIM13 & S & Lys-265-Lys \\
YNL273W & TOF1 & S & Asn-117-Asn \\
\hline
\end{tabular}

*S: synonymous, NS: non-synonymous 


\section{Table 2 Physiological characterization of a 35-deletion, minimal CCM prototrophic S. cerevisiae strain in aerobic bioreactor batch cultures}

S. cerevisiae strains were grown at $\mathrm{pH} 5.0$ and at $30{ }^{\circ} \mathrm{C}$ in aerobic bioreactors on synthetic medium with glucose as sole carbon source. Data are presented as average and standard deviation of 3 biological replicates for S. cerevisiae strains CEN.PK113-7D (naïve reference) and IMX2538 (minimal CCM). Data for S. cerevisiae IMX372 (minimal glycolysis) were recalculated from the raw data of Solis-Escalante et al. (2015) obtained with two biological replicates. Statistical significance with respect to CEN.PK113-7D is represented in bold with an asterisk (two-tailed t-test, equal variances, $\mathrm{P}<0.05$ ).

\begin{tabular}{|c|c|c|c|}
\hline & $\begin{array}{l}\text { CEN.PK113-7D } \\
\text { (Naïve reference) }\end{array}$ & $\begin{array}{l}\text { IMX372 } \\
\text { (Minimal Glycolysis) }\end{array}$ & $\begin{array}{l}\text { IMX2538 } \\
\text { (Minimal CCM) }\end{array}$ \\
\hline \multicolumn{4}{|l|}{ Glucose phase } \\
\hline$\mu_{\max }\left(h^{-1}\right)$ & $0.37 \pm 0.00$ & $0.38 \pm 0.01 *$ & $0.34 \pm 0.00 *$ \\
\hline$q_{s}\left(m_{m o l} g_{D W}{ }^{-1} \cdot h^{-1}\right)$ & $-16.2 \pm 0.2$ & $-15.7 \pm 0.7$ & $-15.4 \pm 0.5$ \\
\hline qEthanol (mmol.g. $\left.\mathrm{Dw}^{-1} \cdot \mathrm{h}^{-1}\right)$ & $23.5 \pm 1.5$ & $23.1 \pm 1.1$ & $23.2 \pm 2.1$ \\
\hline qGlycerol (mmol.g $\left.{ }_{D w}^{-1} \cdot h^{-1}\right)$ & $1.52 \pm 0.05$ & $1.40 \pm 0.02$ & $1.11 \pm 0.05^{*}$ \\
\hline qAcetate (mmol.g $\left.g_{D w^{-1}} \cdot h^{-1}\right)$ & $0.44 \pm 0.03$ & $0.82 \pm 0.04^{*}$ & $0.71 \pm 0.02 *$ \\
\hline $\mathrm{qCO}_{2}\left(\mathrm{mmol} \cdot \mathrm{gDw}^{-1} \cdot \mathrm{h}^{-1}\right)$ & $23.4 \pm 0.2$ & & $22.6 \pm 0.5$ \\
\hline $\mathrm{qO}_{2}\left(\mathrm{mmol} \cdot \mathrm{gDw}^{-1} \cdot \mathrm{h}^{-1}\right)$ & $-6.8 \pm 0.4$ & & $-7.0 \pm 0.2$ \\
\hline$Y_{\text {biomass/glucose }}\left(\right.$ g $_{\text {DW. }}$ gglucose $\left.^{-1}\right)$ & $0.13 \pm 0.00$ & $0.13 \pm 0.00$ & $0.12 \pm 0.00$ \\
\hline $\mathrm{Y}_{\text {ethanol/glucose }}\left(\mathrm{mol} \cdot \mathrm{mol}^{-1}\right)$ & $1.45 \pm 0.09$ & $1.48 \pm 0.01$ & $1.51 \pm 0.09$ \\
\hline$Y_{\text {glycerol/glucose }}\left(\mathrm{mol} \mathrm{mol}^{-1}\right)$ & $0.09 \pm 0.00$ & $0.09 \pm 0.01$ & $0.07 \pm 0.00 *$ \\
\hline $\mathbf{Y}_{\text {acetate/glucose }}\left(\mathrm{mol} \mathrm{mol}^{-1}\right)$ & $0.03 \pm 0.00$ & $0.05 \pm 0.00$ & $0.05 \pm 0.00 *$ \\
\hline \multicolumn{4}{|l|}{ Post-diauxic phase } \\
\hline$\mu_{\max }\left(h^{-1}\right)$ & $0.10 \pm 0.00$ & $0.12 \pm 0.00 *$ & $0.08 \pm 0.01 *$ \\
\hline qEthanol (mmol.g $\left.\mathrm{Dw}^{-1} \cdot \mathrm{h}^{-1}\right)$ & $-3.10 \pm 0.19$ & $-3.93 \pm 0.04 *$ & $-3.07 \pm 0.31$ \\
\hline
\end{tabular}




\section{Table 3 Intracellular metabolite profiles of a 35-deletion, minimal CCM}

\section{prototrophic S. cerevisiae strain in aerobic bioreactor batch cultures}

Intracellular metabolite contents were measured during the mid-exponential glucose phase of aerobic bioreactor batch cultures of S. cerevisiae CEN.PK113-7D (naïve reference) and IMX2538 (minimal CCM) (see Table 2 for other physiological data). Data represent average and standard deviation of data from analyses on three independent cultures for each strain. Fold differences that are statistically significant are indicated in bold with an asterisk (two-tailed t-test, equal variances, $\mathrm{P}<0.05$ ). \# below detection limit.

\begin{tabular}{|c|c|c|c|}
\hline Metabolite & $\begin{array}{l}\text { CEN.PK113-7D } \\
\text { (naïve reference) }\end{array}$ & $\begin{array}{l}\text { IMX2538 } \\
\text { (Minimal CCM) }\end{array}$ & $\begin{array}{l}\text { Fold } \\
\text { difference }\end{array}$ \\
\hline \multicolumn{4}{|c|}{$\mu \mathrm{mol} .(\mathrm{g} \text { biomass dry weight })^{-1}$} \\
\hline \multicolumn{4}{|l|}{ Glycolysis } \\
\hline Glucose 6-phosphate & $4.13 \pm 0.49$ & $5.01 \pm 0.38$ & 1.2 \\
\hline Fructose 6-phosphate & $0.38 \pm 0.06$ & $0.59 \pm 0.08$ & $1.5^{*}$ \\
\hline Fructose 1,6-bisphosphate & $17.53 \pm 0.82$ & $20.52 \pm 0.66$ & $1.2^{*}$ \\
\hline Glyceraldehyde 3-phosphate & $0.15 \pm 0.01$ & $0.19 \pm 0.01$ & $1.3^{*}$ \\
\hline Dihydroxyacetone phosphate & $0.79 \pm 0.13$ & $2.08 \pm 0.66$ & 2.6 \\
\hline 3-Phosphoglycerate & $1.30 \pm 0.12$ & $2.52 \pm 0.28$ & $1.9^{*}$ \\
\hline 2-Phosphoglycerate & $0.16 \pm 0.01$ & $0.17 \pm 0.01$ & 1.0 \\
\hline Phosphoenolpyruvate & $0.25 \pm 0.01$ & $0.12 \pm 0.01$ & $0.5^{*}$ \\
\hline \multicolumn{4}{|l|}{ Trehalose synthesis } \\
\hline Trehalose 6-phosphate & $9.34 \pm 1.18$ & $14.48 \pm 1.67$ & $1.6^{*}$ \\
\hline Trehalose $\mathrm{T}^{\#}$ & $0.20 \pm 0.04$ & $0.41 \pm 0.09$ & $2.1^{*}$ \\
\hline \multicolumn{4}{|l|}{ Pentose phosphate pathway } \\
\hline 6-Phosphoglucononate & $1.01 \pm 0.02$ & $1.11 \pm 0.07$ & 1.1 \\
\hline Ribose 5-phosphate & $0.40 \pm 0.02$ & $0.51 \pm 0.03$ & $1.3^{*}$ \\
\hline Ribulose 5-phosphate & $0.20 \pm 0.03$ & $0.33 \pm 0.06$ & $1.6^{*}$ \\
\hline Xylulose 5-phosphate & $0.43 \pm 0.07$ & $0.72 \pm 0.13$ & 1.7 \\
\hline Sedoheptulose 7-phosphate & $0.50 \pm 0.06$ & $0.54 \pm 0.04$ & 1.1 \\
\hline Erythrose 4-phosphate & $0.004 \pm 0.000$ & $0.005 \pm 0.000$ & $1.3^{*}$ \\
\hline \multicolumn{4}{|l|}{ TCA cycle and Glyoxylate cycle } \\
\hline Citrate & $4.56 \pm 0.65$ & $5.80 \pm 0.98$ & 1.3 \\
\hline Isocitrate & $0.02 \pm 0.00$ & $0.03 \pm 0.00$ & $1.7 *$ \\
\hline$\alpha$-ketoglutarate & $0.29 \pm 0.02$ & $0.67 \pm 0.24$ & 2.3 \\
\hline Succinate & $0.37 \pm 0.03$ & $0.37 \pm 0.12$ & 1.0 \\
\hline Fumarate & $0.10 \pm 0.02$ & $0.18 \pm 0.06$ & 1.8 \\
\hline Malate & $0.60 \pm 0.08$ & $1.03 \pm 0.19$ & $1.7^{*}$ \\
\hline \multicolumn{4}{|l|}{ Nucleotides and cofactors } \\
\hline Energy charge & $0.86 \pm 0.00$ & $0.88 \pm 0.01$ & 1.0 \\
\hline AMP & $0.13 \pm 0.01$ & $0.17 \pm 0.01$ & $1.3^{*}$ \\
\hline ADP & $1.55 \pm 0.08$ & $1.43 \pm 0.11$ & 0.9 \\
\hline ATP & $5.50 \pm 0.16$ & $5.58 \pm 0.21$ & 1.0 \\
\hline
\end{tabular}


bioRxiv preprint doi: https://doi.org/10.1101/2021.08.24.457526; this version posted August 26, 2021. The copyright holder for this preprint (which was not certified by peer review) is the author/funder, who has granted bioRxiv a license to display the preprint in perpetuity. It is made available under aCC-BY-NC-ND 4.0 International license.

\begin{tabular}{llll}
\hline UDP & $0.23 \pm 0.01$ & $0.22 \pm 0.05$ & 0.9 \\
UTP & $1.61 \pm 0.06$ & $1.57 \pm 0.10$ & 1.0 \\
GDP & $0.27 \pm 0.02$ & $0.21 \pm 0.07$ & 0.8 \\
GTP & $1.58 \pm 0.06$ & $1.49 \pm 0.15$ & 0.9 \\
CDP & $0.13 \pm 0.02$ & $0.15 \pm 0.01$ & 1.1 \\
CTP & $0.84 \pm 0.02$ & $0.82 \pm 0.03$ & 1.0 \\
NADH & $0.18 \pm 0.02$ & $0.15 \pm 0.02$ & 0.8 \\
NAD $^{+}$ & $85.43 \pm 1.90$ & $91.82 \pm 2.64$ & $\mathbf{1 . 1}^{*}$ \\
NADP $^{+}$ & $8.49 \pm 0.67$ & $6.87 \pm 0.25$ & $\mathbf{0 . 8}$ \\
Acetyl-CoA & $5.80 \pm 0.18$ & $5.38 \pm 0.34$ & 0.9 \\
FAD & $0.87 \pm 0.12$ & $0.54 \pm 0.18$ & 0.6 \\
\hline
\end{tabular}

